Review Article

\title{
Foeniculum vulgare Mill: A Review of Its Botany, Phytochemistry, Pharmacology, Contemporary Application, and Toxicology
}

\author{
Shamkant B. Badgujar, Vainav V. Patel, and Atmaram H. Bandivdekar \\ Department of Biochemistry, National Institute for Research in Reproductive Health, ICMR, Jehangir Merwanji Street, Parel, \\ Mumbai, Maharashtra 400 012, India
}

Correspondence should be addressed to Shamkant B. Badgujar; sham83badgujar@gmail.com

Received 11 February 2014; Revised 26 May 2014; Accepted 9 June 2014; Published 3 August 2014

Academic Editor: Ronald E. Baynes

Copyright (c) 2014 Shamkant B. Badgujar et al. This is an open access article distributed under the Creative Commons Attribution License, which permits unrestricted use, distribution, and reproduction in any medium, provided the original work is properly cited.

\begin{abstract}
Foeniculum vulgare Mill commonly called fennel has been used in traditional medicine for a wide range of ailments related to digestive, endocrine, reproductive, and respiratory systems. Additionally, it is also used as a galactagogue agent for lactating mothers. The review aims to gather the fragmented information available in the literature regarding morphology, ethnomedicinal applications, phytochemistry, pharmacology, and toxicology of Foeniculum vulgare. It also compiles available scientific evidence for the ethnobotanical claims and to identify gaps required to be filled by future research. Findings based on their traditional uses and scientific evaluation indicates that Foeniculum vulgare remains to be the most widely used herbal plant. It has been used for more than forty types of disorders. Phytochemical studies have shown the presence of numerous valuable compounds, such as volatile compounds, flavonoids, phenolic compounds, fatty acids, and amino acids. Compiled data indicate their efficacy in several in vitro and in vivo pharmacological properties such as antimicrobial, antiviral, anti-inflammatory, antimutagenic, antinociceptive, antipyretic, antispasmodic, antithrombotic, apoptotic, cardiovascular, chemomodulatory, antitumor, hepatoprotective, hypoglycemic, hypolipidemic, and memory enhancing property. Foeniculum vulgare has emerged as a good source of traditional medicine and it provides a noteworthy basis in pharmaceutical biology for the development/formulation of new drugs and future clinical uses.
\end{abstract}

\section{Introduction}

Foeniculum vulgare is the oldest valid name within the genus Foeniculum for the plant designated by Karsten as Foeniculum Foeniculutn. However, according to the international rules of nomenclature, the binomial name Foeniculum vulgare was not validly published by Hill in his reference [1] for the reason that he did not consistently adopt the binomial system of nomenclature. In accordance with the international rules as adopted at Cambridge, the name Foeniculum vulgare must be accredited to Philip Miller, who first validly published it in the eighth edition of his "Gardeners Dictionary" in 1768. From then on, the name of this plant is written as Foeniculum vulgare Mill. It is a medicinal plant belonging to the Umbelliferae (Apiaceae) family, known and used by humans since antiquity, due to its flavor. It was cultivated in almost every country [2]. It is universally known as Fennel and is known by more than 100 names (Table 1). It is a traditional and popular herb with a long history of use as a medicine. A series of studies showed that $F$. vulgare effectively controls numerous infectious disorders of bacterial, fungal, viral, mycobacterium, and protozoal origin [3-7]. It has antioxidant, antitumor, chemopreventive, cytoprotective, hepatoprotective, hypoglycemic, and oestrogenic activities [8-12]. Some of the publications stated that $F$. vulgare has a special kind of memory-enhancing effect and can reduce stress [13]. Animal experiments and limited clinical trials suggest that chronic use of $F$. vulgare is not harmful. Fennel maybe consumed daily, in the raw form as salads and snacks, stewed, boiled, grilled, or baked in several dishes and even used in the preparation of herbal teas or spirits. A diet with desired quantity of fennel could bring potential health 
benefits due to its valuable nutritional composition with respect to presence of essential fatty acids [14]. In recent years, increased interests in improvement of agricultural yield of fennel due to its medicinal properties and essential oil content has encouraged cultivation of the plant on large scale.

Research on F. vulgare with current technology has been conducted all over the world. All the available literature on F. vulgare was compiled from electronic databases such as Academic Journals (including high impact, nonimpact, and nonindexed journals), Ethnobotany, Google Scholar, Scopus link, PubMed, Science Direct, Web of Science, and library search. A review of the literature from 2001 to 2005 shows only $20 \%$ reports published on F. vulgare which increased to about $38 \%$ from 2006 to 2010 . Briefly, in these 10 years a total of 89 claims appeared in the literature on various aspects of F. vulgare. It is important to note that about $39 \%$ of reports (61 articles) were collected from recent three years, that is, 2011 to 2013 (Figure 1). Some of the earlier published reviews of this plant included medicinal properties and phytochemistry [15-20], but few of them appear in all these reviews. However, there is a need for an inclusive review that bridges the gaps between traditional uses of fennel and its in vitro studies. The present review attempts to collate the available information on the botany, nationwise common vernacular names, cultivation (propagation), nutritive value, and traditional/contemporary as well as allied applications, phytochemistry, pharmacology, and toxicity of F. vulgare. We hope that this review may provide scientific basis that explains the ethnophytopharmacological role of F. vulgare in order to facilitate and guide future research. In particular, we aimed to answer the following questions. (1) What information is available on the traditional uses, botany, phytochemistry, and toxicity of F. vulgare? (2) What pharmacological studies were performed on this plant and how do they validate its traditional uses? (3) What is the future for F. vulgare?

1.1. Taxonomy. Kingdom: Plantae, division: Tracheophyta, subdivision: Spermatophytina, class: Magnoliopsida, order: Apiales, family: Apiaceae, genus: Foeniculum, species: vulgare, and botanical name: Foeniculum vulgare Mill.

1.2. Botanical Description. Fennel is an ancient seasonal herb. The fennel plant originated in the southern Mediterranean region and through naturalization and cultivation it grows wild throughout the Northern, Eastern, and Western hemispheres, specifically in Asia, North America, and Europe. It is cultivated in fields and also grows wild. The herb was well-known to the ancient Egyptians, Romans, Indians, and Chinese. The Romans grew it for its aromatic seeds and the edible fleshy shoots are still a very common vegetable in southern Italy [21]. Emperor Charlemagne was known to have encouraged its cultivation in Central Europe. It is an indispensable ingredient in modern French and Italian cooking. All parts of the plant are aromatic and can be used in many ways.

F. vulgare is an upright, branching perennial herb (Figure 2(a)) with soft, feathery, almost hair-like foliage
TABLE 1: Vernacular names of Foeniculum vulgare.

\begin{tabular}{|c|c|}
\hline $\begin{array}{l}\text { Region/language/system of } \\
\text { medicine }\end{array}$ & Local name \\
\hline Alto, Bolivia & Hinojo \\
\hline Arabic & Bisbas, razianaj \\
\hline Aymara, Kechua & Inuju \\
\hline Balikesir, Turkey & $\begin{array}{l}\text { Arapsaci, rezene, malatura, } \\
\text { hullebe }\end{array}$ \\
\hline Basque & Mieloi \\
\hline Bengali (Indian language) & Mauri, pānmourī \\
\hline Bosnia & Komorač \\
\hline Brazil & Endro, erva-doce, funcho \\
\hline Catalan & Fenoll, fonoll \\
\hline Central Serbia & Morac \\
\hline Chinese & Hui xiang, xiao hui xiang \\
\hline Czech & Fenykl \\
\hline $\begin{array}{l}\text { Dalmatia (southern Croatia), } \\
\text { Poland }\end{array}$ & $\begin{array}{l}\text { Komorač, koromač, kumurač, } \\
\text { morač, moroč, morača, Koper } \\
\text { wloski }\end{array}$ \\
\hline Danish & Almindelig fennikel, fennikel \\
\hline Denmark & Almindelig \\
\hline Dutch & Venkel \\
\hline English & $\begin{array}{l}\text { Bitter fennel, common fennel, } \\
\text { sweet fennel, wild fennel }\end{array}$ \\
\hline France & Fenouille \\
\hline French & Fenouil \\
\hline Germany & $\begin{array}{l}\text { Fenchel, fenchle, bitterfenchel, } \\
\text { wilder fenchel, dunkler fenchel, }\end{array}$ \\
\hline Guerrero, Mexico & Hinojo \\
\hline Gujarati (Indian language) & Hariyal, variyali \\
\hline Haryana, India & Saunf \\
\hline Hindi (Indian language) & $\begin{array}{l}\text { Badi, badishep, bari saunf, badi } \\
\text { saunf, saunp, saunf, sonp, sont }\end{array}$ \\
\hline Italy & $\begin{array}{l}\text { Finucchio, finucchiello, } \\
\text { finochietto, finocchiella, } \\
\text { fenùcciu, fenucéttu-sarvègu }\end{array}$ \\
\hline Jammu and Kashmir, India & Saunf \\
\hline Japanese & $\begin{array}{l}\text { Fenneru, uikyou, uikyou, } \\
\text { shouikya }\end{array}$ \\
\hline Java, Indonesia & Adas \\
\hline Jordan & Shomar \\
\hline Kallawaya & Jinuchchu \\
\hline Kannada & $\begin{array}{l}\text { Badi sopu, badisepu, sabbasige, } \\
\text { dodda sopu, dodda jirige }\end{array}$ \\
\hline Korea & Sohoehyang \\
\hline Laotian & Phaksi \\
\hline Latin & Foeniculum, maratrum \\
\hline Loja, Ecuador & Hinojo \\
\hline Majorcan area & Fonoll \\
\hline Middle Navarra & Hinojo, cenojo \\
\hline
\end{tabular}


TABle 1: Continued.

\begin{tabular}{ll}
\hline $\begin{array}{l}\text { Region/language/system of } \\
\text { medicine }\end{array}$ & Local name \\
\hline Marathi (Indian language) & Badishep, badiiśep, shoap \\
Nepalese & Madesi sauf \\
North Iran & Badian \\
North Portugal & Funcho \\
Norway & Fenikkel \\
Norwegian & Fennikel \\
Pakistan & Sonef, saunf \\
Peninsula, Spain & Hinojo \\
Persian & Razianeh \\
Polish & Fenkuł, koper włoski \\
Portuguese & Funcho \\
Rajasthan, India & Sanuf \\
Sanskrit (Indian language) & Madhurika, shatapushpa \\
Slovenian & Sladki komarček \\
Somali Region, Ethiopia & Kamon \\
South Europe & Fennel \\
South Africa & Vinkel, fennel \\
Spanish & Hinojo, hinojo amargo, fenoll, \\
Swedish & Follo, millua \\
Tamil (Indian language) & Perun siragum, shombu, sohikire \\
Telugu (Indian language) & Peddajilakurra, sopu \\
Thai & Phak chi, phak chi duen ha, phak \\
Uttarakhand, India & chi lom, thian klaep, yira \\
\hline & \\
\hline & \\
Sadesoppu
\end{tabular}

growing upto $6.6 \mathrm{ft} .(2 \mathrm{~m})$ tall. This plant looks similar to dill. It is typically grown in vegetable and herb gardens (Figure 2(f)) for its anise-flavored foliage and seeds, both of which are commonly harvested for use in cooking. It is erect and cylindrical, bright green, and smooth as to seem polished, with multiple branched leaves (Figure 2(c)) cut into the finest of segments. The leaves grow upto $40 \mathrm{~cm}$ long; they are finely dissected, with the ultimate segments filiform (threadlike), about $0.5 \mathrm{~mm}$ wide. The bright golden flowers, produced in large, flat terminal umbels, with thirteen to twenty rays, bloom in July and August (Figure 2(d)).

Foliage. Stem striate, leaves 3-4 pinnate, segments filiform, upto $1.6 \mathrm{in}$. $(4 \mathrm{~cm})$ long; leaf bases sheathing. It has a green, sleek, and slippery stem with upright stiff branches and much divided leaves in linear segments (Figure 2(b)). Rays are 530 numbers with $0.39-2.4$ inches $(1-6 \mathrm{~cm})$ long. Flowers are small, yellow, and found in large flat-topped umbels (Figure 2(d)). Fruits are oblong to ovoid with $0.12-0.2$ inches (3-5 mm) long and $1.5-2.0 \mathrm{~mm}$ broad (Figure 2(e)). The stylopodium persists on the fruit. The fruits are elongated and have strong ribs. The most esteemed fennel seeds vary from three to five lines in length and are elliptical, slightly curved, and somewhat obtuse at the ends (Figure 3(a)).

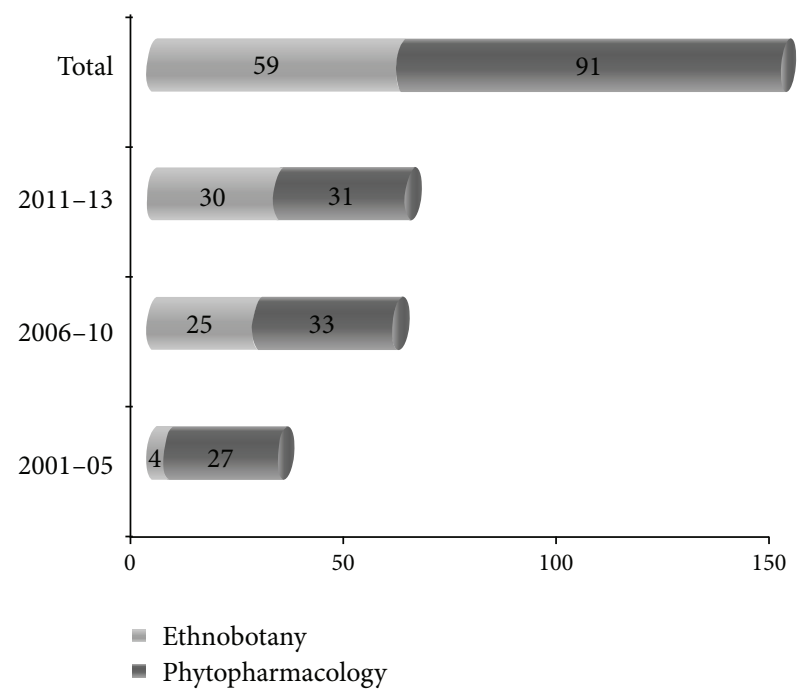

FIGURE 1: Research papers in different aspects especially traditional or ethnobotanical knowledge, phytochemistry, pharmacological, and various biological activities of Foeniculum vulgare. (Papers were collected via electronic databases such as Academic Journals, Ethnobotany, Google Scholar, PubMed, and Science Direct.)

They are greenish-yellow, the colour of hay, from which the term fennel is derived. Wild fruits are short, dark coloured and blunt at their ends, and have a less agreeable flavour and odour than those of sweet fennel. Seeds ripen from September to October. This plant can reproduce from crown or root fragments but freely reproduces from seed.

1.3. Chemical Composition and Nutritional Value of Fennel. Foeniculum vulgare is widely grown for its edible fruit or seeds. These are sweet and dry; a fully ripe specimen is an exquisite fruit. The fruit is often dried for later use and this dried fruit called fennel is a major item of commerce. Table 2 lists the nutrient composition of fennel (USDA data). Fennels are one of the highest plant sources of potassium, sodium, phosphorus, and calcium. According to USDA data for the Mission variety, fennels are richest in dietary fiber and vitamins, relative to human needs. They have smaller amounts of many other nutrients.

Table 3 summarizes the chemical composition and the nutritional value [14] of different parts of fennel, namely, shoots, leaves, stems, and inflorescence. Leaves and stems show the highest moisture content (76.36 and $77.46 \mathrm{~g} / 100 \mathrm{~g}$, resp.), while inflorescence exhibits the lowest content $(71.31 \mathrm{~g} / 100 \mathrm{~g})$. Carbohydrates are the most abundant macronutrients in all the parts and range from 18.44 to $22.82 \mathrm{~g} / 100 \mathrm{~g}$. Proteins, reducing sugars, and fats are the less abundant macronutrients; proteins varied between $1.08 \mathrm{~g} / 100 \mathrm{~g}$ in stems and $1.37 \mathrm{~g} / 100 \mathrm{~g}$ in inflorescences. The inflorescences and stems revealed the highest fat content $(1.28 \mathrm{~g} / 100 \mathrm{~g})$ and reducing sugar content $(1.49 \mathrm{~g} / 100 \mathrm{~g})$, respectively, amongst all the parts of fennel. On the basis of the proximate analysis, it can be calculated that a fresh portion of $100 \mathrm{~g}$ of these parts yields, on average, $94 \mathrm{Kcal}$ of 


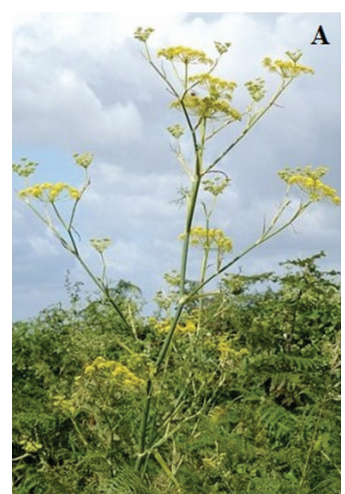

(a)

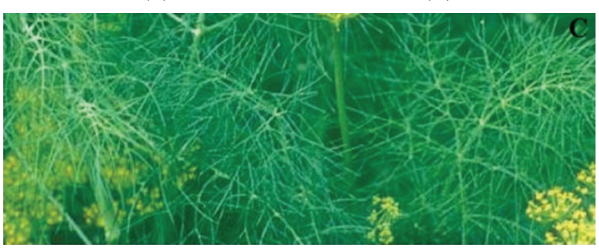

(c)

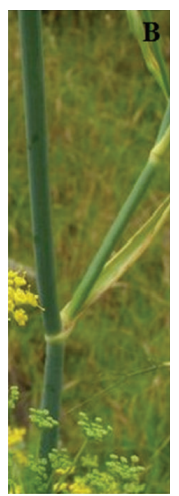

(b)

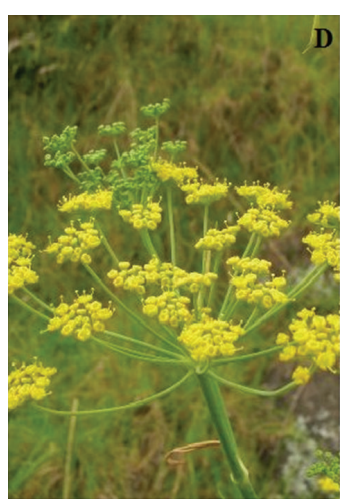

(d)

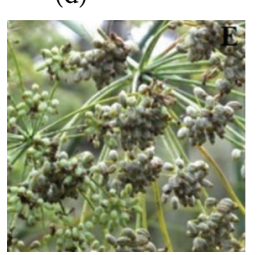

(e)

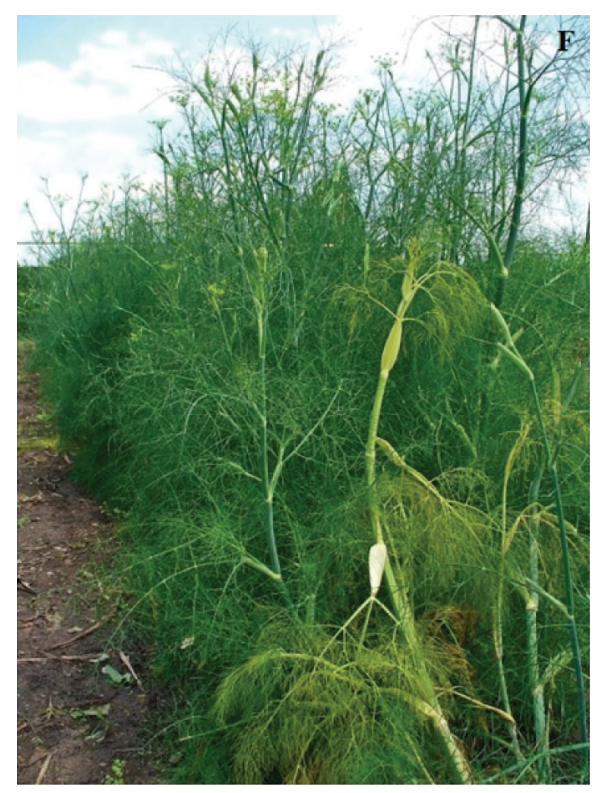

(f)

FIGURE 2: Foeniculum vulgare Mill (a) in its natural habitat; (b) stem; (c) leaves; (d) inflorescences and flowers; (e) fruits; and (f) population of F. vulgare Mill.

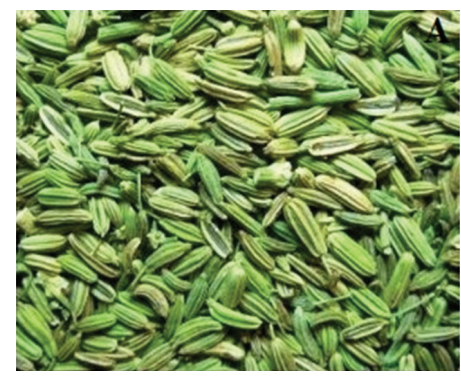

(a)

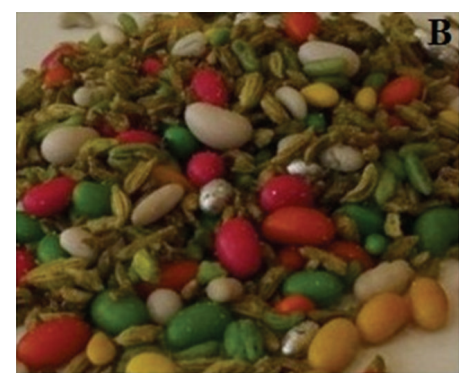

(b)

FIGURE 3: Normal fennel seeds (a) and sugar coated and uncoated fennel seeds (b) used in mukhwas.

energy. The highest values were obtained for inflorescences, while leaves and stems gave the lowest energy contribution.

About twenty-one fatty acids were identified and quantified from the above mentioned parts of fennel (Table 3 ). These are caproic acid, caprylic acid, capric acid, undecanoic acid, lauric acid, myristic acid, myristoleic acid, pentadecanoic acid, palmitic acid, heptadecanoic acid, stearic acid, oleic acid, linoleic acid, $\alpha$-linolenic acid, arachidic acid, eicosanoic acid, cis-11,14-eicosadienoic acid, cis-11,14,17-eicosatrienoic acid + heneicosanoic acid, behenic acid, tricosanoic acid, and lignoceric acid. Thus, Barros and his coworker conclude polyunsaturated fatty acids (PUFA) to be the main group of fatty acids present in all the fennel parts. On the other hand Vardavas and his coworker reported monounsaturated fatty acids (MUFA) as the main group of fatty acids in fennel [22]. Nevertheless, unsaturated fatty acids (UFA) range from $66 \%$ to $80 \%$ and predominate over saturated fatty acids [14]. The highest concentration of n-3 fatty acids was found in fennel leaves, while the lowest concentration was found in inflorescences. The ratio of $\omega 6$ to $\omega 3$ fatty acids has an important role in the human diet. The highest levels of n-3 fatty acids found in leaves contributed to its lowest ratio of $\omega 6$ to $\omega 3$ fatty acids. The lowest levels of n-3 fatty acids found in inflorescences contributed to its highest ratio of $\omega 6$ to $\omega 3$ fatty acids.

Fennels have smaller amounts of many other nutrients. On a weight basis, fennels contain more calcium (49 mg/ $100 \mathrm{~g}$ ) as compared with apples $(7.14 \mathrm{mg} / 100 \mathrm{~g})$, bananas $(3.88 \mathrm{mg} / 100 \mathrm{~g})$, dates $(25.0 \mathrm{mg} / 100 \mathrm{~g})$, grapes $(10.86 \mathrm{mg} /$ $100 \mathrm{~g})$, orange $(40.25 \mathrm{mg} / 100 \mathrm{~g})$, prunes $(18.0 \mathrm{mg} / 100 \mathrm{~g})$, raisins $(40.0 \mathrm{mg} / 100 \mathrm{~g})$, and strawberries $(14.01 \mathrm{mg} / 100 \mathrm{~g})$. Phenolics are an important constituent of fruit quality because of their contribution to the taste, colour, and nutritional properties of fruit. Amongst the phenolics analyzed in the fruit of this plant are neochlorogenic acid (1.40\%), chlorogenic acid $(2.98 \%)$, gallic acid $(0.169 \%)$, chlorogenic acid (6.873\%), caffeic acid (2.960\%), p-coumaric acid $(4.325 \%)$, ferulic acid-7-o-glucoside (5.223\%), quercetin-7-o-glucoside 
TABLE 2: Nutrients found in dried fennel (USDA, USA).

\begin{tabular}{|c|c|}
\hline Composition & Quantity (Per $100 \mathrm{~g}$ ) \\
\hline \multicolumn{2}{|l|}{ Proximates } \\
\hline Moisture & $90.21 \mathrm{~g}$ \\
\hline Energy & $31 \mathrm{kcal}$ \\
\hline Protein & $1.24 \mathrm{~g}$ \\
\hline Total lipid (fat) & $0.2 \mathrm{~g}$ \\
\hline Carbohydrate & $7.3 \mathrm{~g}$ \\
\hline Total dietary fiber & $3.1 \mathrm{~g}$ \\
\hline Sugars & $3.93 \mathrm{~g}$ \\
\hline \multicolumn{2}{|l|}{ Minerals } \\
\hline Calcium, $\mathrm{Ca}$ & $49 \mathrm{mg}$ \\
\hline Iron, $\mathrm{Fe}$ & $0.73 \mathrm{mg}$ \\
\hline Magnesium, Mg & $17 \mathrm{mg}$ \\
\hline Phosphorus, $\mathrm{P}$ & $50 \mathrm{mg}$ \\
\hline Potassium, K & $414 \mathrm{mg}$ \\
\hline Sodium, $\mathrm{Na}$ & $52 \mathrm{mg}$ \\
\hline Zinc, $\mathrm{Zn}$ & $0.2 \mathrm{mg}$ \\
\hline \multicolumn{2}{|l|}{ Vitamins } \\
\hline Vitamin C & $12 \mathrm{mg}$ \\
\hline Thiamin B-1 & $0.01 \mathrm{mg}$ \\
\hline Riboflavin B-2 & $0.032 \mathrm{mg}$ \\
\hline Niacin B-3 & $0.64 \mathrm{mg}$ \\
\hline Vitamin B-6 & $0.047 \mathrm{mg}$ \\
\hline Folate & $27 \mu \mathrm{g}$ \\
\hline Vitamin A & $48 \mu \mathrm{g}$ \\
\hline Vitamin E & $0.58 \mathrm{mg}$ \\
\hline Vitamin K & $62.8 \mu \mathrm{g}$ \\
\hline \multicolumn{2}{|l|}{ Lipids } \\
\hline Fatty acids, total saturated & $0.09 \mathrm{~g}$ \\
\hline Fatty acids, total monounsaturated & $0.068 \mathrm{~g}$ \\
\hline Fatty acids, total polyunsaturated & $0.169 \mathrm{~g}$ \\
\hline \multicolumn{2}{|l|}{ Essential amino acids } \\
\hline Leucine & $0.63 \mathrm{~g}$ \\
\hline Isoleucine & $0.73 \mathrm{~g}$ \\
\hline Phenylalanine & $0.45 \mathrm{~g}$ \\
\hline Tryptophane & $0.53 \mathrm{~g}$ \\
\hline \multicolumn{2}{|l|}{ Nonessential amino acid } \\
\hline Glycine & $0.55 \mathrm{~g}$ \\
\hline Proline & $0.53 \mathrm{~g}$ \\
\hline
\end{tabular}

(3.219\%), ferulic acid (3.555\%), 1,5 dicaffeoylquinic acid $(4.095 \%)$, hesperidin $(0.203 \%)$, cinnamic acid $(0.131 \%)$, rosmarinic acid (14.998\%), quercetin (17.097\%), and apigenin (12.558\%) [23].

Thus, as a typical, seasonal fresh fruit, fennels are an important constituent of the regional diet of Europe and other regions. Different varieties of fennel parts are widely used in many of the cooking dishes all over world (Table 4). Shoots, tender leaves, and stems are chewed and sucked due to their exquisite aniseed flavor. All these parts are also commonly used as vegetables. They are added raw to salads, stewed with beans and chickpeas, used to stuff fish for grilling, and placed in soups and bread bouillons. Besides seasoning, fennel is used to preserve food. Flowering stems, sugar, and honey macerating in brandy produce a highly valorized spirit. Herbal teas prepared with fresh tender or dried flowering stems are consumed chilled or hot, depending on the season. F. vulgare is famous for its essential oil. The characteristic anise odour of $F$. vulgare, which is due to its essential oil, makes it an excellent flavoring agent in baked goods, meat and fish dishes, ice-cream, and alcoholic beverages. The culinary uses of fennel are so diverse/widespread that it has been exported from country to country for centuries [14].

\section{Traditional and Contemporary Uses}

Foeniculum vulgare has been extensively used in traditional medicine for a wide range of ailments. Fennel is used in various traditional systems of medicine like in the Ayurveda, Unani, Siddha, in the Indian, and Iranian traditional systems of alternative and balancing medicine [20]. Its stem, fruit, leaves, seeds, and whole plant itself are medicinally used in different forms in the treatment of a variety of diseased conditions. The preparation methods, uses, and application of F. vulgare are well documented in the common ethnobotanical literature [24-32]. Table 5 lists the ethnomedicinal uses of F. vulgare for 43 different types of ailments in Bolivia, Brazil, Ecuador, Ethiopia, India, Iran, Italy, Jordan, Mexico, Pakistan, Portugal, Serbia, South Africa, Spain, Turkey, and USA [28, 29, 33-44]. It is used to treat simple ailments (e.g., cough/cold, cuts) to very complicated ailments (e.g., kidney ailments, cancer). It also has a wide range of veterinary uses $([45,46]$ see Table 4). F. vulgare is used in many parts of the world for the treatment of a number of diseases, for example, abdominal pains, antiemetic, aperitif, arthritis, cancer, colic in children, conjunctivitis, constipation, depurative, diarrhea, dieresis, emmenagogue, fever, flatulence, gastralgia, gastritis, insomnia, irritable colon, kidney ailments, laxative, leucorrhoea, liver pain, mouth ulcer, and stomachache (Table 5).

In addition to its medicinal uses, aerial parts, namely, leaf, stem, and fruit/seed of F. vulgare, are extensively used as galactagogues not only for increasing the quantity and quality of milk but also for improving the milk flow of breastfeeding mothers [32, 34, 37, 47]. From ancient times, fennel seeds have been used as an ingredient for removing any foul smell of the mouth [48]. The natural light green dye obtained from leaves is used in cosmetics, for coloring of textiles/wooden materials and as food colorant. Yellow and brown color dyes are obtained by combining the flowers and leaves of fennel [49]. In Portugal, Italy, Spain, and India, the stem, fruit, leaves, seeds, and whole plant are used as a vegetable [3, 9, $48,50,51]$. Sugar coated and uncoated fennel seeds are used in mukhwas (Mouth freshener) (Figure 3(b)). In many parts of India and Pakistan, roasted fennel seeds are consumed as mukhwas (Mouth freshener). Mukhwas is a colorful aftermeal mouth freshener or digestive aid. It can be made of various seeds and nuts but often found with fennel seeds, anise seeds, coconut, and sesame seeds. They are sweet in flavor and highly aromatic due to the presence of sugar and the addition of various essential oils. The seeds can be savory, coated in sugar, and brightly colored. 
TABLE 3: Nutrient content of different parts of Foeniculum vulgare.

\begin{tabular}{|c|c|c|c|c|}
\hline \multirow{2}{*}{ Composition } & \multicolumn{4}{|c|}{ Contents } \\
\hline & Leaves & Inflorescences & Stems & Shoots \\
\hline Moisture $^{\mathrm{a}}$ & $76.36 \pm 0.33$ & $71.31 \pm 4.01$ & $77.46 \pm 1.03$ & $73.88 \pm 0.83$ \\
\hline $\mathrm{Ash}^{\mathrm{a}}$ & $3.43 \pm 0.04$ & $3.23 \pm 0.02$ & $1.62 \pm 0.12$ & $2.39 \pm 0.02$ \\
\hline $\mathrm{Fat}^{\mathrm{a}}$ & $0.61 \pm 0.16$ & $1.28 \pm 0.28$ & $0.45 \pm 0.07$ & $0.49 \pm 0.05$ \\
\hline Protein $^{\mathrm{a}}$ & $1.16 \pm 0.03$ & $1.37 \pm 0.05$ & $1.08 \pm 0.00$ & $1.33 \pm 0.04$ \\
\hline Carbohydrates $^{\mathrm{a}}$ & $18.44 \pm 0.06$ & $22.82 \pm 3.06$ & $19.39 \pm 0.65$ & $21.91 \pm 0.55$ \\
\hline Fructose $^{a}$ & $0.49 \pm 0.05$ & $1.10 \pm 0.04$ & $1.49 \pm 0.04$ & $1.51 \pm 0.06$ \\
\hline Glucose $^{a}$ & $0.76 \pm 0.12$ & $2.94 \pm 0.11$ & $3.43 \pm 0.20$ & $4.71 \pm 0.15$ \\
\hline Sucrose $\mathrm{a}^{\mathrm{a}}$ & $0.04 \pm 0.00$ & $0.03 \pm 0.00$ & nd & $0.35 \pm 0.06$ \\
\hline Reducing sugars $^{\mathrm{a}}$ & $0.72 \pm 0.04$ & $1.20 \pm 0.19$ & $1.49 \pm 0.29$ & $1.14 \pm 0.10$ \\
\hline$\omega 3$ fatty acid ${ }^{b}$ & $43.72 \pm 0.36$ & $17.69 \pm 0.01$ & $23.04 \pm 1.30$ & $36.96 \pm 0.51$ \\
\hline$\omega 6$ fatty acid ${ }^{\mathrm{b}}$ & $23.25 \pm 0.07$ & $38.94 \pm 0.23$ & $38.22 \pm 0.68$ & $39.99 \pm 0.68$ \\
\hline$\omega 6 / \omega 3$ & $0.53 \pm 0.00$ & $2.20 \pm 0.01$ & $1.66 \pm 1.12$ & $1.08 \pm 0.03$ \\
\hline $\mathrm{C} 6: 0^{\mathrm{b}}$ & $0.02 \pm 0.00$ & $0.41 \pm 0.02$ & $0.19 \pm 0.01$ & $0.06 \pm 0.00$ \\
\hline $\mathrm{C} 8: 0^{\mathrm{b}}$ & $0.08 \pm 0.00$ & $0.37 \pm 0.01$ & $0.48 \pm 0.03$ & $0.33 \pm 0.00$ \\
\hline $\mathrm{C} 10: 0^{\mathrm{b}}$ & $0.04 \pm 0.00$ & $0.09 \pm 0.00$ & $0.13 \pm 0.01$ & $0.06 \pm 0.00$ \\
\hline $\mathrm{C} 11: 0^{\mathrm{b}}$ & $0.25 \pm 0.02$ & $0.29 \pm 0.01$ & $0.04 \pm 0.00$ & $0.07 \pm 0.00$ \\
\hline $\mathrm{C} 12: 0^{\mathrm{b}}$ & $0.31 \pm 0.02$ & $0.43 \pm 0.06$ & $0.11 \pm 0.01$ & $0.21 \pm 0.02$ \\
\hline $\mathrm{C} 14: 0^{\mathrm{b}}$ & $1.43 \pm 0.01$ & $1.68 \pm 0.10$ & $0.49 \pm 0.06$ & $0.75 \pm 0.03$ \\
\hline $\mathrm{C} 14: 1^{\mathrm{b}}$ & $0.61 \pm 0.04$ & $0.28 \pm 0.02$ & $0.37 \pm 0.04$ & $0.17 \pm 0.03$ \\
\hline $\mathrm{C} 15: 0^{\mathrm{b}}$ & $0.17 \pm 0.00$ & $0.35 \pm 0.03$ & $0.41 \pm 0.04$ & $0.18 \pm 0.00$ \\
\hline $\mathrm{C} 16: 0^{\mathrm{b}}$ & $20.15 \pm 0.09$ & $23.89 \pm 0.07$ & $25.43 \pm 0.00$ & $12.78 \pm 0.09$ \\
\hline $\mathrm{C} 17: 0^{\mathrm{b}}$ & $0.74 \pm 0.00$ & $0.58 \pm 0.02$ & $0.61 \pm 0.04$ & $0.24 \pm 0.02$ \\
\hline $\mathrm{C} 18: 0^{\mathrm{b}}$ & $1.61 \pm 0.08$ & $2.62 \pm 0.04$ & $1.99 \pm 0.06$ & $1.53 \pm 0.08$ \\
\hline$C 18: \ln 9 c^{b}$ & $4.35 \pm 0.37$ & $5.05 \pm 0.00$ & $4.35 \pm 0.52$ & $2.55 \pm 0.33$ \\
\hline $\mathrm{C} 18: 2 \mathrm{n} 6 \mathrm{c}^{\mathrm{b}}$ & $23.25 \pm 0.07$ & $38.94 \pm 0.23$ & $38.22 \pm 0.68$ & $39.99 \pm 0.68$ \\
\hline $\mathrm{C} 18: 3 \mathrm{n} 3^{\mathrm{b}}$ & $43.55 \pm 0.40$ & $17.55 \pm 0.0$ & $22.86 \pm 1.31$ & $36.84 \pm 0.52$ \\
\hline $\mathrm{C} 20: 0^{\mathrm{b}}$ & $0.56 \pm 0.00$ & $1.78 \pm 0.06$ & $0.84 \pm 0.03$ & $1.06 \pm 0.09$ \\
\hline $\mathrm{C} 20: 1 \mathrm{c}^{\mathrm{b}}$ & nd & $0.26 \pm 0.03$ & $0.06 \pm 0.00$ & nd \\
\hline $\mathrm{C} 20: 2 \mathrm{c}^{\mathrm{b}}$ & $0.08 \pm 0.01$ & $0.31 \pm 0.01$ & $0.14 \pm 0.00$ & $0.38 \pm 0.07$ \\
\hline $\mathrm{C} 20: 3 \mathrm{n} 3+\mathrm{C} 21: 0^{\mathrm{b}}$ & $0.16 \pm 0.02$ & $0.15 \pm 0.01$ & $0.19 \pm 0.00$ & $0.12 \pm 0.01$ \\
\hline $\mathrm{C} 22: 0^{\mathrm{b}}$ & $0.77 \pm 0.04$ & $1.52 \pm 0.04$ & $1.20 \pm 0.03$ & $1.12 \pm 0.02$ \\
\hline $\mathrm{C} 23: 0^{\mathrm{b}}$ & $0.82 \pm 0.13$ & $1.89 \pm 0.11$ & $0.68 \pm 0.01$ & $0.36 \pm 0.15$ \\
\hline $\mathrm{C} 24: 0^{\mathrm{b}}$ & $1.03 \pm 0.04$ & $1.58 \pm 0.02$ & $1.21 \pm 0.02$ & $1.20 \pm 0.08$ \\
\hline Total SFA ${ }^{b}$ & $27.99 \pm 0.02$ & $37.47 \pm 0.25$ & $33.81 \pm 0.06$ & $19.95 \pm 0.12$ \\
\hline Total MUFA ${ }^{\mathrm{b}}$ & $4.96 \pm 0.40$ & $5.59 \pm 0.13$ & $4.78 \pm 0.57$ & $2.72 \pm 0.36$ \\
\hline Total PUFA ${ }^{\mathrm{b}}$ & $67.05 \pm 0.42$ & $56.94 \pm 0.12$ & $61.41 \pm 0.62$ & $77.33 \pm 0.24$ \\
\hline Energy $^{c}$ & $83.90 \pm 1.34$ & $108.23 \pm 10.37$ & $85.91 \pm 3.02$ & $97.37 \pm 2.44$ \\
\hline
\end{tabular}

${ }^{\mathrm{a}}$ Nutrients composition $(\mathrm{g} / 100 \mathrm{~g}),{ }^{\mathrm{b}} \omega 3$ and $\omega 6$ and fatty acid content (percent), and ${ }^{\mathrm{c}}$ energetic value (Kcal/100 g) of the different parts of fennel. nd: not detected. Values are expressed as mean $\pm \mathrm{SD}, n=3$ experiments in each group [14].

\section{Phytochemistry}

Phytochemical research carried out on Foeniculum vulgare has led to the isolation of fatty acids, phenolic components, hydrocarbons, volatile components, and few other classes of secondary metabolites from its different parts (Figure 4). Mostly these phytochemicals are found in essential oil (Table 6). Some of the phytoconstituents of F. vulgare were find application as coloring and antiaging agents $[49,50]$. They also have noteworthy biological and pharmacological activities (Table 7).
3.1. Volatile Compounds. Table 6 summarizes the volatile compounds present in the essential oil of $F$. vulgare. The anise odor of F. vulgare is due to its essential oil content. It makes an excellent flavoring agent in various types of food and food related products. The essential oil of fennel has been reported to contain more than 87 volatile compounds [51-57]. The accumulation of these volatile compounds inside the plant is variable, appearing practically in any of its parts, namely, roots, stem, shoots, flowers, and fruits $[58,59]$. The molecular structures of major volatile components of $F$. vulgare seed essential oil have been illustrated in Figure 4. 
TABLE 4: Uses of Foeniculum vulgare as a food ingredient as reported in the literature.

\begin{tabular}{|c|c|c|c|c|}
\hline Sr. number & Region/Nation & Local name & Part used and edible application. & References \\
\hline 1 & Campania, Italy & $\begin{array}{l}\text { Finucchio, } \\
\text { finucchiello, } \\
\text { finochietto }\end{array}$ & Stem is used as an aromatizer for pickled olives. & {$[125]$} \\
\hline 2 & Campania, Italy & $\begin{array}{l}\text { Finocchiella, } \\
\text { fenùcciu }\end{array}$ & Seed is employed in preparation of salted meats. & {$[125]$} \\
\hline 3 & Spain & Hinojo, Fenoll & $\begin{array}{l}\text { Tender leaves and stems, raw as a snack, are used in } \\
\text { salads or stewed. }\end{array}$ & {$[126]$} \\
\hline 4 & Spain & Fiallo, millau & $\begin{array}{l}\text { Aerial part or seeds used for seasoning olives, as } \\
\text { preservative for dry figs, and for preparing herbal tea or } \\
\text { liqueur. }\end{array}$ & {$[126]$} \\
\hline 5 & $\begin{array}{l}\text { Trás-os-Montes } \\
\text { (Northeast } \\
\text { Portuguese) }\end{array}$ & $\begin{array}{l}\text { Fialho, fionho, } \\
\text { erva-doce }\end{array}$ & $\begin{array}{l}\text { Shoots, tender leaves, and stems used in snacks, salads, } \\
\text { soups, stews, and spices. } \\
\text { Flowering stems used in beverages, spirits, and spices. } \\
\text { Stems used as brochettes and herbal teas. } \\
\text { Seeds used as spices, flavour for cakes, biscuits, and } \\
\text { sweets, and chestnuts. }\end{array}$ & {$[14]$} \\
\hline 6 & $\begin{array}{l}\text { Arrábida and } \\
\text { Açor (Center } \\
\text { Portuguese) }\end{array}$ & Funcho, erva-doce & $\begin{array}{l}\text { Seeds used as flavour for cakes and pastries and for } \\
\text { cooking chestnuts. }\end{array}$ & {$[14]$} \\
\hline 7 & $\begin{array}{l}\text { Alentejo and } \\
\text { Algarve (South } \\
\text { Portuguese) }\end{array}$ & $\begin{array}{l}\text { Funcho, fialho, } \\
\text { funcho-doce, } \\
\text { funcho-amargo }\end{array}$ & $\begin{array}{l}\text { Shoots, tender leaves, and stems are fried with eggs, } \\
\text { used in omelettes, used in fish stuff, stewed with } \\
\text { different kinds of beans and chickpeas, and used in fish } \\
\text { and bread bouillons, soups, and sauces. } \\
\text { Tender leafy stems are used in grilled fish and fish } \\
\text { dishes in general. } \\
\text { Seeds are used as spices, flavour for cakes, bread, and } \\
\text { biscuits, and chestnuts. } \\
\text { Whole plant used in olives brines, figs preserves, and } \\
\text { for aromatizing brandy. }\end{array}$ & {$[14]$} \\
\hline 8 & $\begin{array}{c}\text { Jammu and } \\
\text { Kashmir, India }\end{array}$ & Saunf & $\begin{array}{l}\text { The fruits with other ingredients are given to the animal } \\
\text { if it stops taking food during diarrhea. }\end{array}$ & {$[46]$} \\
\hline 9 & Liguria, Italy & Fenucéttu-sarvègu & $\begin{array}{l}\text { Aerial parts of plant mixed with shoots of Clematis and } \\
\text { Rubus used as food integrator for sheep. }\end{array}$ & {$[45]$} \\
\hline
\end{tabular}

Guillén and Manzanos [60] investigated the yield and composition of the volatile components found in the pentane extracts of leaves, stems, and seeds of F. vulgare. They identified a total of 37 volatile compounds from pentane extracts of above mentioned parts of fennel by using gas chromatography (GC) and gas chromatography-mass spectrometry (GC-MS) techniques. In the supercritical $\mathrm{CO}_{2}$ (SC$\mathrm{CO}_{2}$ ) seed extracts of fennel, a total of 28 compounds were identified with major compounds being trans-anethole (68.6-75.0\%), fenchone (8.40-14.7\%), and methylchavicol (5.09-9.10\%) whereas only 19 compounds were detected from hydrodistilled oil of fennel [52]. Fang et al. [53] characterizes 76 volatile components in the essential oil of $F$. vulgare with the help of three advanced techniques, namely, headspace solvent microextraction followed by gas chromatography-mass spectrometry (HSME-GC-MS), solid phase microextraction(SPME-) GC-MS, and steam distillation- (SD-) GC-MS methods. In 2007 Tognolini et al. investigated the chemical composition of essential oil of fennel. GC/MS study revealed a total of 18 compounds present in it with anethole being the most abundant [55]. A comparative profile of occurrence of monoterpene hydrocarbons, oxygenated monoterpenes, and phenylpropanoids with respect to various maturity stages (immature, premature, mature, and fully mature) of the fruit of F. vulgare was reported by Telci et al. [56]. They concluded that the content of essential oil decreases with increasing maturity. A total of 28 components of the essential oil were identified, accounting for $98.0 \%$ of the total oil. The principal compound in the essential oil was trans-anethole (72.2\%) followed by estragole (7.6\%), d-limonene (6.8\%), and fenchone, that is, $3.9 \%$ [61]. Overall, 60 compounds representing $90.1-98.7 \%$ of the essential oil were identified by GC and GC/MS in the two cultivars of fennel, namely, Aurelio and Sparta cocultivars. The major constituent of the essential oils is trans-anethole (59.8-90.4\%). In addition, the fennel essential oils also contains minor amounts of various constituents as limonene (0.1-21.5\%), neophytadiene (0-10.6\%), (E)-phytol (0.1-6.0\%), exo-fenchyl acetate $(0.3-$ $3.8 \%)$, estragole $(0.1-2.5 \%)$, and fenchone, that is, $0.1-3.1 \%$ [62]. In addition, Zoubiri et al. [57] summarized the comparative profile of volatile compounds found in different varieties of fennel from different countries such as Estonia, Norway, Austria, Moldova, and Turkey. The chemical composition of the Algerian F. vulgare seed oil was different as compared with 
TABLE 5: Traditional and contemporary applications of Foeniculum vulgare.

\begin{tabular}{|c|c|c|c|c|}
\hline Sr. number & Ailment/use & Part/preparation used & Locality & References \\
\hline 1 & Mouth ulcer & Tender leaves, chewed and stuck on ulcer & Basilicata, Italy & [33] \\
\hline 2 & Aperitif & Tender parts-raw or boiled & Rome, Italy & {$[32]$} \\
\hline 3 & Gum disorder & $\begin{array}{l}\text { Fruit and seed, used as a mouth wash for gum } \\
\text { disorder }\end{array}$ & Central Serbia & [35] \\
\hline 4 & Insomnia & Infusion of tea leaf & Brazil & {$[31]$} \\
\hline \multirow{2}{*}{5} & \multirow{2}{*}{ Constipation } & Seeds, decoction & South Europe & {$[127]$} \\
\hline & & Seeds mixed with sugar & $\begin{array}{l}\text { Jammu and } \\
\text { Kashmir, India }\end{array}$ & {$[36]$} \\
\hline 6 & Cancer & Leaf and flower, aqueous infusion, drink & Loja, Ecuador & {$[128]$} \\
\hline 7 & Conjunctivitis & Leaf and flower, aqueous infusion, drink & Loja, Ecuador & [128] \\
\hline 8 & Gastritis & Leaf, flower, aqueous infusion, drink & Loja, Ecuador & [128] \\
\hline 9 & Diuresis & Root and seed, decoction & $\begin{array}{l}\text { Miami, Florida, } \\
\text { USA }\end{array}$ & {$[42]$} \\
\hline \multirow{3}{*}{10} & \multirow{3}{*}{ Abdominal pains } & Each plant part, decoction & Rome, Italy & {$[30]$} \\
\hline & & Leaf and seeds, infusion & $\begin{array}{l}\text { Northern Badia, } \\
\text { Jordan, }\end{array}$ & {$[39]$} \\
\hline & & Leaves, paste & Manisa, Turkey & {$[43]$} \\
\hline 11 & Cold & Fruits and floral tops, decoction & Rome, Italy & {$[30]$} \\
\hline 12 & Refreshing & Roots/whole plant, decoction & Rome, Italy & {$[30]$} \\
\hline 13 & Swollen stomach & Leaves, decoction with a little honey & Rome, Italy & [129] \\
\hline 14 & Hair grow & Seed oil & Middle Navarra & {$[130]$} \\
\hline 15 & Antiemetic & Fruit, simple powder & $\begin{array}{l}\text { Northeastern } \\
\text { Majorcan area }\end{array}$ & {$[47]$} \\
\hline 16 & $\begin{array}{l}\text { Antihypertensive } \\
\text { and Anti- } \\
\text { cholesterolemic }\end{array}$ & Leaf directly chewed & $\begin{array}{l}\text { north-eastern } \\
\text { Majorcan area }\end{array}$ & {$[47]$} \\
\hline 17 & Depurative & Leaf and stem, comestible & $\begin{array}{l}\text { Iberian Peninsula, } \\
\text { Spain }\end{array}$ & {$[40]$} \\
\hline 18 & Hypnotic & Seed, leaf, and stem, infusion and edible & North Iran & {$[24]$} \\
\hline \multirow{2}{*}{19} & \multirow{2}{*}{ Diarrhoea } & Seeds, roots, and fresh leaves & Northern Portugal & {$[28]$} \\
\hline & & $\begin{array}{l}\text { Seeds grounded with Root tubers of Hemidesmus } \\
\text { indicus and the paste taken with jaggery twice a day } \\
\text { for three days }\end{array}$ & $\begin{array}{l}\text { Bhandara, } \\
\text { Maharashtra, India }\end{array}$ & {$[131]$} \\
\hline \multirow{2}{*}{20} & \multirow{2}{*}{ Kidney ailments } & Aerial part, infusion & Alto, Bolivia & {$[34]$} \\
\hline & & Seed, decoction & $\begin{array}{l}\text { Gujranwala, } \\
\text { Pakistan }\end{array}$ & {$[132]$} \\
\hline 21 & Colic in children & Leaf and fruit, infusion & Brazil & {$[133]$} \\
\hline 22 & Irritable colon & Leaf and seeds, infusion & $\begin{array}{l}\text { Northern Badia, } \\
\text { Jordan, }\end{array}$ & {$[39]$} \\
\hline 23 & Gastralgia & Leaf, decoction & southern Spain & {$[29]$} \\
\hline 24 & Purgative & Seed, infusion and edible & $\begin{array}{l}\text { Gujranwala, } \\
\text { Pakistan }\end{array}$ & {$[132]$} \\
\hline 25 & Laxative & Seed, infusion and edible & $\begin{array}{l}\text { Gujranwala, } \\
\text { Pakistan }\end{array}$ & {$[132]$} \\
\hline 26 & Liver pain & Seed & $\begin{array}{l}\text { Pernambuco, } \\
\text { Northeast Brazil }\end{array}$ & {$[133]$} \\
\hline 27 & Mosquitocidal & Root boiled and drunk as tea & $\begin{array}{l}\text { Somali Region, } \\
\text { Ethiopia }\end{array}$ & {$[41]$} \\
\hline 28 & Arthritis & Leaf, an infusion made from the leaves is drunk & South Africa & {$[37]$} \\
\hline 29 & Fever & Leaf, an infusion made from the leaves is drunk & South Africa & {$[37]$} \\
\hline
\end{tabular}


TABle 5: Continued.

\begin{tabular}{|c|c|c|c|c|}
\hline Sr. number & Ailment/use & Part/preparation used & Locality & References \\
\hline 30 & Fat deduction & Green fruit is chewed to reduce fat & South Africa & {$[37]$} \\
\hline 31 & Leucorrhoea & $\begin{array}{l}\text { A mixture of its } 100 \mathrm{~g} \text { seed powder, } 200 \mathrm{~g} \text { seed } \\
\text { powder of Papaver somniferum, } 100 \mathrm{~g} \text { fruit powder of } \\
\text { Coriander sativum, and } 200 \mathrm{~g} \text { of sugar is prepared } \\
\text { and } 50 \mathrm{~g} \text { of this mixture is taken by the tribal ladies } \\
\text { early in the morning }\end{array}$ & Rajasthan, India & {$[26]$} \\
\hline 32 & $\begin{array}{l}\text { Problem of } \\
\text { repeated abortions }\end{array}$ & $\begin{array}{l}\text { Mixture of its } 50 \mathrm{~g} \text { seed powder, } 50 \mathrm{~g} \text { fruit powder of } \\
\text { Trapa natans, and } 50 \mathrm{~g} \text { sugar is given daily to } \\
\text { pregnant ladies }\end{array}$ & Rajasthan, India & {$[26]$} \\
\hline \multirow{7}{*}{33} & \multirow{7}{*}{ Digestive system } & Fruits, decoction & Basilicata, Italy & {$[33]$} \\
\hline & & Seed, decoction (drink one tea cup after food) & Balikesir, Turkey & {$[134]$} \\
\hline & & Whole plant & $\begin{array}{l}\text { Western cape of } \\
\text { South Africa }\end{array}$ & {$[135]$} \\
\hline & & Fruit, powder for digestive ailments & $\begin{array}{l}\text { Middle, West, and } \\
\text { South Bosnia }\end{array}$ & {$[136]$} \\
\hline & & Seeds, decoction & South Europe & {$[127]$} \\
\hline & & Seeds, roots, and fresh leaves & Northern Portugal. & {$[28]$} \\
\hline & & Seed, decoction & Southern Spain & [29] \\
\hline \multirow{5}{*}{34} & \multirow{5}{*}{ Carminative } & Tender parts, raw or boiled & Rome, Italy & {$[32]$} \\
\hline & & Whole plant & $\begin{array}{l}\text { Western cape of } \\
\text { South Africa }\end{array}$ & {$[135]$} \\
\hline & & Seeds, decoction & South Europe & {$[127]$} \\
\hline & & Seed, leaf, and stem, infusion and edible & North Iran & {$[24]$} \\
\hline & & Leaves and/or fruits & South Africa & {$[27]$} \\
\hline \multirow{5}{*}{35} & \multirow{5}{*}{ Diuretic } & Tender parts, raw or boiled & Rome, Italy & {$[32]$} \\
\hline & & Whole plant & $\begin{array}{l}\text { Western cape of } \\
\text { South Africa }\end{array}$ & {$[135]$} \\
\hline & & Seeds, decoction & South-Europe & {$[127]$} \\
\hline & & Seeds, roots, and fresh leaves & Northern Portugal. & {$[28]$} \\
\hline & & Leaf, an infusion made from the leaves is drunk & South Africa & {$[37]$} \\
\hline \multirow{3}{*}{36} & \multirow{3}{*}{ Emmenagogue } & Aerial part, raw with carrot & Rome, Italy & {$[32]$} \\
\hline & & Fruit, simple powder & $\begin{array}{l}\text { North-eastern } \\
\text { Majorcan area }\end{array}$ & {$[47]$} \\
\hline & & Seed & Haryana, India & {$[137]$} \\
\hline \multirow{4}{*}{37} & \multirow{4}{*}{$\begin{array}{l}\text { Milk stimulant in } \\
\text { pregnant women } \\
\text { (Galactagogue) }\end{array}$} & Leaf, an infusion made from the leaves is drunk & South Africa & {$[37]$} \\
\hline & & Fruits, as condiment or chewed & Rome, Italy & {$[32]$} \\
\hline & & Fruit, simple powder & $\begin{array}{l}\text { north-eastern } \\
\text { Majorcan area }\end{array}$ & {$[47]$} \\
\hline & & Aerial part-infusion & Alto, Bolivia & {$[34]$} \\
\hline \multirow{2}{*}{38} & \multirow{2}{*}{ Gingival wound } & Fruit-paste & Uttarakhand, India & {$[138]$} \\
\hline & & Whole plant, decoction & Andalusia, Spain & {$[29]$} \\
\hline \multirow{4}{*}{39} & \multirow{4}{*}{$\begin{array}{l}\text { Eye blurry and } \\
\text { itching }\end{array}$} & Aerial parts, inhaled into eyes & Balikesir, Turkey & {$[134]$} \\
\hline & & Seeds, roots, and leaves & Northern Portugal & {$[28]$} \\
\hline & & Seed, infusion, edible & $\begin{array}{l}\text { Gujranwala, } \\
\text { Pakistan }\end{array}$ & {$[132]$} \\
\hline & & Leaves and/or fruits & South Africa & {$[27]$} \\
\hline \multirow{3}{*}{40} & \multirow{3}{*}{ Cough } & Whole plant, oral infusion & Guerrero, Mexico & {$[44]$} \\
\hline & & Whole plant, decoction & Southern Spain & {$[29]$} \\
\hline & & Whole plant & $\begin{array}{l}\text { Western cape of } \\
\text { South Africa }\end{array}$ & {$[135]$} \\
\hline
\end{tabular}


TABLE 5: Continued.

\begin{tabular}{|c|c|c|c|c|}
\hline Sr. number & Ailment/use & Part/preparation used & Locality & References \\
\hline \multirow{4}{*}{41} & \multirow{4}{*}{ Stomachache } & Whole plant, oral infusion & Guerrero, Mexico & {$[44]$} \\
\hline & & Fruit & Middle Navarra & {$[130]$} \\
\hline & & Seed decoction is used against stomach ache & Liguria, Italy & {$[45]$} \\
\hline & & Seed, leaf, and stem-infusion, edible & North Iran & {$[24]$} \\
\hline \multirow{2}{*}{42} & \multirow{2}{*}{ Stress removal } & \multirow{2}{*}{ Apical shoots is used as sedative for children } & Liguria, Italy & {$[45]$} \\
\hline & & & $\begin{array}{c}\text { Southern Punjab, } \\
\text { Pakistan }\end{array}$ & {$[25]$} \\
\hline \multirow{3}{*}{43} & \multirow{3}{*}{ Flatulence } & Leaf and fruit, infusion & Brazil & {$[133]$} \\
\hline & & Leaf and seeds, infusion & $\begin{array}{c}\text { Northern Badia, } \\
\text { Jordan, }\end{array}$ & {$[39]$} \\
\hline & & Fresh fruit, decoction & $\begin{array}{l}\text { North Bengal, } \\
\text { India }\end{array}$ & {$[38]$} \\
\hline
\end{tabular}

Turkish [51, 56], Serbian [52], Indian [54], and Chinese [53] fennels. The hexane extracts of fennel were analyzed by GCMS and 78 compounds were identified from these extracts; the major compounds were identified as 1,3-benzenediol, 1methoxycyclohexene, o-cymene, sorbic acid, 2-hydroxy-3methyl-2-cyclopenten-1-one, estragole, limonene-10-ol, and 3-methyl-2-cyclopenten-1-one [63]. Diao et al. [64] identify a total of 28 components by GC and GC/MS from fennel oil, representing $95.8 \%$ of the total amount. Trans-Anethole (68.53\%), a phenylpropanoid, was found to be the main component, followed by estragole $(10.42 \%)$ with limonene (6.24\%), fenchone (5.45\%), and others as minor components.

3.2. Flavonoids. Flavonoids are generally considered as an important category of antioxidants in the human diet. Flavonoids are abundant in the plants of Apiaceae family. It has been reported that the presence of flavonol glycosides in fennel species is related to its morphological heterogeneity and variation. Total flavonoid content of hydroalcoholic extracts is about $12.3 \pm 0.18 \mathrm{mg} / \mathrm{g}$. Flavonoids like eriodictyol-7-rutinoside, quercetin-3-rutinoside, and rosmarinic acid have been isolated from F. vulgare [65]. Amongst the flavonoids present in F. vulgare, the most prevalent are quercetin-3-glucuronide, isoquercitrin, quercetin3-arabinoside, kaempferol-3-glucuronide and kaempferol-3arabinoside, and isorhamnetin glucoside [66]. Quercetin-3$O$-galactoside, kaempferol-3-O-rutinoside, and kaempferol3-O-glucoside have also been reported to occur in the aqueous extract of F. vulgare [67]. The flavonoids like isorhamnetin 3-O- $\alpha$-rhamnoside, quercetin, and kaempferol were also isolated from the ethyl acetate extract, whereas quercetin 3-O-rutinoside, kaempferol 3-O-rutinoside, and quercetin 3-O- $\beta$-glucoside were isolated from the methanol extract. These flavonoids exhibit remarkable antinociceptive and antiinflammatory activity [68]. Further, quercetin, rutin, and isoquercitrin were reported to have the immunomodulatory activities [69].

3.3. Phenolic Compounds. There has been a growing interest in phenolic components of fruits and vegetables, which may promote human health or lower the risk of disease. Aqueous extract of fennel fruits are rich in phenolic compounds. Many of them have antioxidant activities and hepatoprotective properties. The phenolic compounds present in $F$. vulgare are considered to be associated with the prevention of diseases possibly induced by oxidative stress such as cardiovascular diseases, cancer, and inflammation. These phenolic compounds have received tremendous attention among nutritionists, food scientists, and consumers due to their role in human health. Fennel has been reported to contain hydroxyl cinnamic acid derivatives, flavonoid glycosides, and flavonoid aglycones [67]. The methanolic extract of fennel seeds contains rosmarinic acid, chlorogenic acids as major phenolic compounds (14.9\% and 6.8\%, resp.), and quercetin and apigenin as the major flavonoids $(17.1 \%$ and $12.5 \%$, resp.). Also, the total phenolic compounds in fennel methanol extract were higher than the flavonoid compounds [23]. F. vulgare has been reported to contain phenolic acids like 3-O-caffeoylquinic acid, 4-O-caffeoylquinic acid, 5-Ocaffeoylquinic acid, 1,3-O-di-caffeoylquinic acid, 1,4-O-dicaffeoylquinic acid, and 1,5-O-di-caffeoylquinic acid [65]. Two compounds A and B were isolated and characterized for the first time from the wild fennel and identified as 3,4-dihydroxyphenethylalchohol-6-O-caffeoyl- $\beta$-Dglucopyranoside and $3^{\prime}, 8^{\prime}$-binaringenin, respectively. The total phenolic and flavonoid contents of wild fennel $(2.4 \%$ and $1.2 \%$ resp.) were less as compared to cultivated fennel $(3.1 \%$ and $1.6 \%$, resp.) [70].

\section{Pharmacological Activities}

Foeniculum vulgare is officially noted in Ayurvedic Pharmacopoeia as an important part of polyherbal formulations in the treatment of different diseases and disorders. A number of biological-pharmacological studies have been undertaken to evaluate the indigenous uses of $F$. vulgare. Few extracts of $F$. vulgare and isolated compounds have been evaluated for several activities, namely, antiaging, antiallergic, anticolitic, antihirsutism, anti-inflammatory, antimicrobial and antiviral, antimutagenic, antinociceptive, antipyretic, antispasmodic, 
<smiles>COc1cc2ccc(=O)oc2cc1O</smiles>

Scopoletin (1)

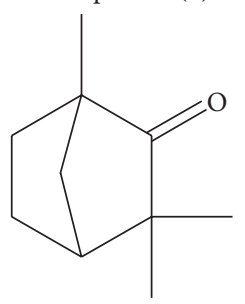

Fenchone (4)<smiles>COc1c2ccoc2cc2oc(=O)ccc12</smiles>

Bergapten (2)

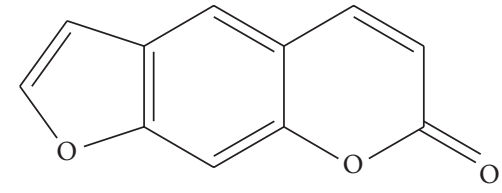

Psoralen (3)

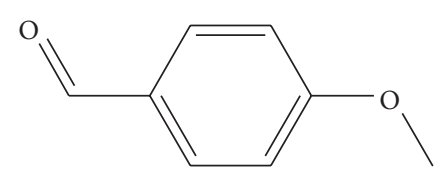

para-Anisaldehyde (5)

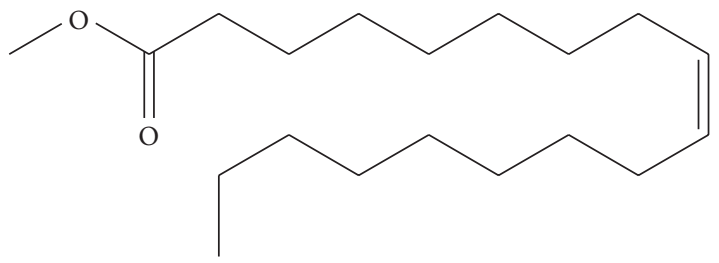

(z)-9-Octadecenoic (6)<smiles>COc1c(/C=C/C=O)cc2c(c1OC)OCO2</smiles>

Dillapional (7)<smiles>C=CC(=C)CCC=C(C)C</smiles><smiles>C=C(C)[C@H]1CC=C(C)CC1</smiles><smiles>C=C(C)CCOc1c2occc2cc2ccc(=O)oc12</smiles><smiles>C=CCc1cc2c(c(OCC)c1OC)OCO2</smiles>

Dillapiol (9)<smiles>CC1=CCC(C(C)(C)O)CC1</smiles>

Terpineol (11)

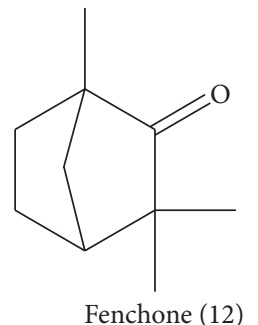

Fenchone (12)

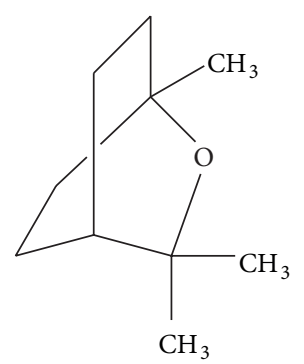

1,8-Cineole (13)

Beta-myrcene (14)<smiles>COc1c2ccoc2cc2oc(=O)ccc12</smiles>

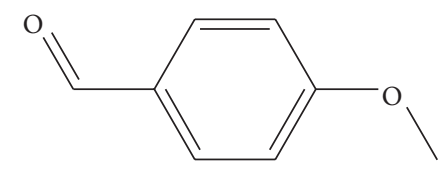

para-Anisaldehyde (16)<smiles>COc1ccc(/C=C\c2ccccc2)cc1</smiles>

Photoanethole (17)

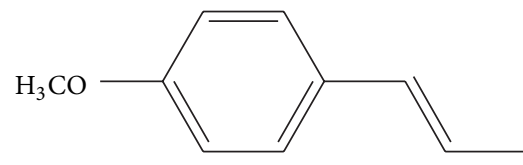

trans-Anethole (18)

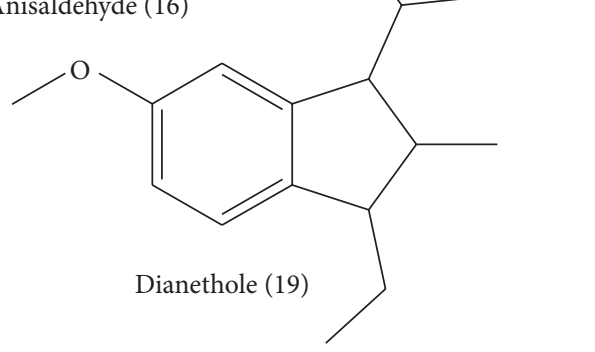

(a)

Figure 4: Continued. 
<smiles>C/C(=C\CO)CCC[C@H](C)CCC[C@H](C)CCCC(C)C</smiles>

(E)-Phytol (20)<smiles>CCCCCC/C=C/C=C/C=O</smiles><smiles>C=CC=CC(C)C(C)c1ccccc1C</smiles><smiles>C=C(C)/C=C/c1ccccc1</smiles><smiles>C=CC(=C)CCCC(C)CCCC(C)CCCC(C)C</smiles><smiles>C=CCc1ccc(OC)cc1</smiles><smiles>Oc1cccc(O)c1</smiles>
1,3-Benzenediol (26)

2-Hydroxy-3-methyl2-cyclopenten-1-one (24)<smiles>COC1=CCCCC1</smiles><smiles>C/C=C/C=C/C(=O)O</smiles>

3,4-Dihydroxyphenethylalchohol-6-O-caffeoyl-beta-D-glucopyranoside (34)

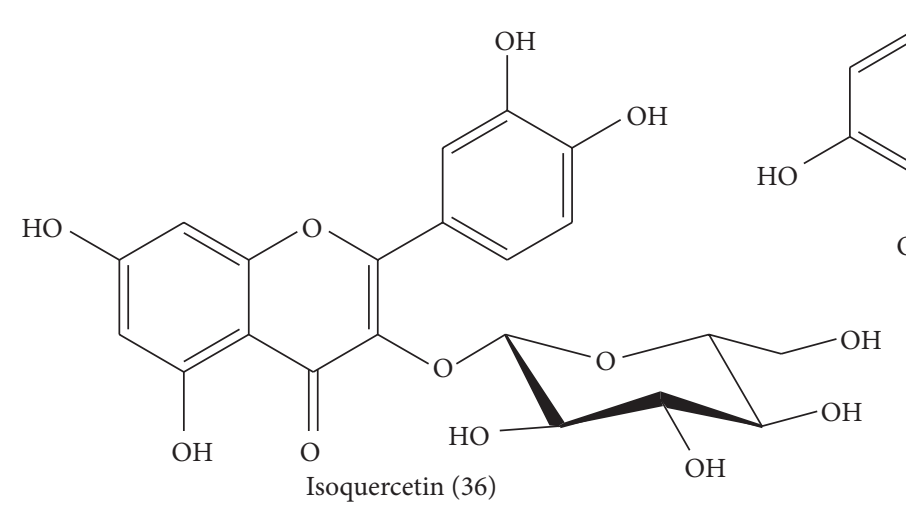

(b)

FIGURE 4: Continued. 
<smiles>CCC=Cc1ccc(O)c(O)c1</smiles>

Caffeoyl

3-O-Caffeoylquinic acid (38)

4-O-Caffeoylquinic acid (39)

5-O-Caffeoylquinic acid (40)

1,3-O-Di-caffeoylquinic acid (41)

1,4-O-Di-caffeoylquinic acid (42)

1,5-O-Di-caffeoylquinic acid (43)

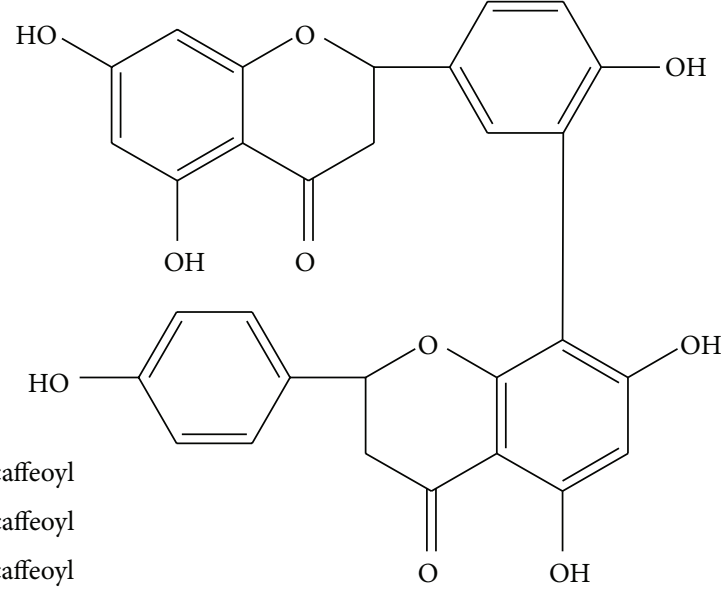

$3^{\prime}, 8^{\prime}$-Binaringenin (44)

$R_{2}=R_{4}=H ;=R_{1}=R_{3}=$ caffeoyl

$\mathrm{R}_{2}=\mathrm{R}_{3}=\mathrm{H} ;=\mathrm{R}_{1}=\mathrm{R}_{4}=$ caffeoyl

$\mathrm{R}_{1}=\mathrm{R}_{3}=\mathrm{R}_{4}=\mathrm{H} ; \mathrm{R}_{2}=$ caffeoyl

$\mathrm{R}_{1}=\mathrm{R}_{2}=\mathrm{R}_{4}=\mathrm{H} ; \mathrm{R}_{3}=$ caffeoy

$\mathrm{R}_{1}=\mathrm{R}_{2}=\mathrm{R}_{3}=\mathrm{H} ; \mathrm{R}_{4}=$ caffeoyl

$\mathrm{R}_{3}=\mathrm{R}_{4}=\mathrm{H} ;=\mathrm{R}_{1}=\mathrm{R}_{2}=$ caffeoyl<smiles>[R1]Oc1c(-c2ccc(O)c([R])c2)oc2cc(O)cc(O)c2c1=O</smiles>

$\mathrm{HO}$<smiles>C=C(/C=C/C)c1oc2cc(I)cc(O)c2c(=O)c1O</smiles>

Quercetin-3-glucuronide (52)

Kaempferol-3-glucuronide (53)

$\mathrm{R}=\mathrm{H}$

Quercetin-3-O-glucoside (45)

Quercetin-3-O-galactoside (46)

Quercetin-3-O-arabinoside (47)

$\mathrm{R}=\mathrm{OH} ; \mathrm{R}_{1}=$ glucose

$\mathrm{R}=\mathrm{OH} ; \mathrm{R}_{1}=$ glucose

$\mathrm{R}=\mathrm{OH} ; \mathrm{R}_{1}=$ arabinose

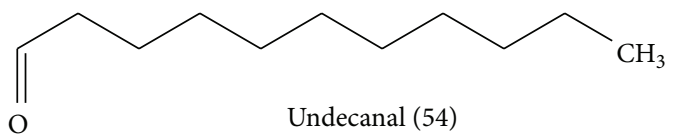

Quercetin-3-O-rutinoside (48)

Kaempferol-3-O-glucoside (49)

$\mathrm{R}=\mathrm{OH} ; \mathrm{R}_{1}=$ glucose-rhamnose

$\mathrm{R}=\mathrm{H} ; \mathrm{R}_{1}=$ glucose

$\mathrm{R}=\mathrm{H} ; \mathrm{R}_{1}=$ arabinose

Kaempferol-3-O-arabinoside (50)

$\mathrm{R}=\mathrm{H} ; \mathrm{R}_{1}=$ glucose-rhamnose

Kaempferol-3-O-rutinoside (51)

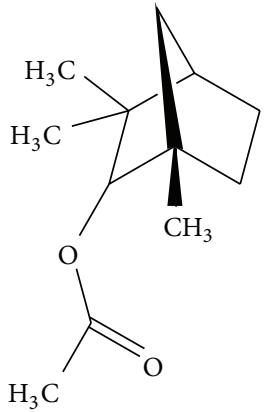

Exo-fenchyl acetate (55)<smiles>COc1c(/C=C/C=O)cc2c(c1OC)OCO2</smiles>

Dillapional (56)

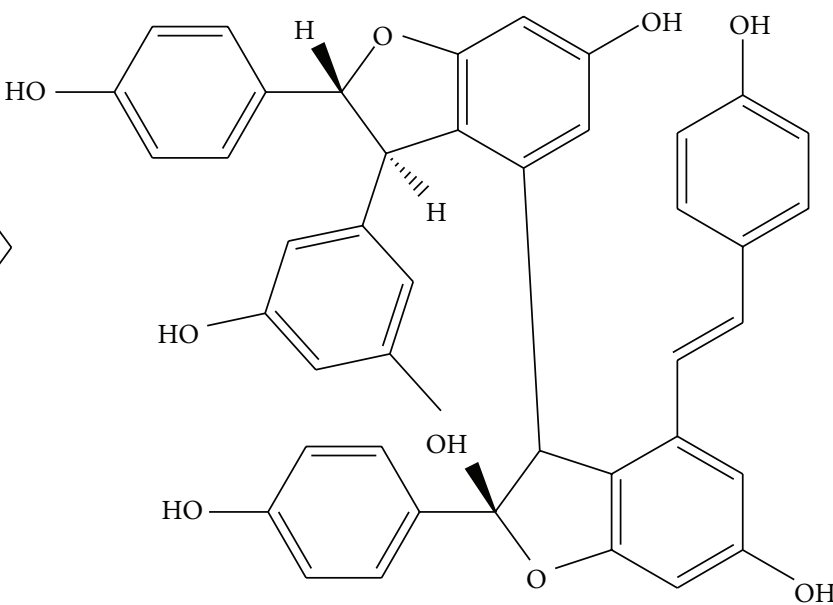

cis-Miyabenol C (57)

(c)

FIgURE 4: Continued. 
<smiles>C[C@@H]1O[C@H](OC[C@H]2O[C@H](Oc3cc(O)c4c(c3)OC(c3ccc(O)c(O)c3)CC4=O)[C@@H](O)[C@H](O)[C@H]2O)[C@H](O)[C@@H](O)[C@H]1O</smiles>

Eriodictyol-7-rutinoside (58)<smiles>C=C(OC)C1CC=C(C)CC1</smiles>

Isorhamnetin-3-O-glucoside (60)

(d)

FIGURE 4: Chemical structures of various phytoconstituents isolated from Foeniculum vulgare.

antistress, antithrombotic, anxiolytic, apoptotic, cardiovascular, chemomodulatory action, cytoprotection and antitumor, cytotoxicity, diuretic, estrogenic properties, expectorant, galactogenic, gastrointestinal effect, hepatoprotective, human liver cytochrome P450 3A4 inhibitory, hypoglycemic, hypolipidemic, memory-enhancing property, nootropic, and oculohypotensive activities $[11,13,20,50,68,71-90]$. Table 8 summarizes the pharmacological studies undertaken on $F$. vulgare and reported in the literature. A brief review of the same is as follows.

4.1. Antimicrobial and Antiviral Activities. Foeniculum vulgare has been used as an ethnic remedy for the cure of numerous infectious disorders of bacterial, fungal, viral, and mycobacterial origin. Several studies have been carried out in the past validating its antimicrobial, antimycobacterial,, and antiviral potential (summarized in the Table 9). Duško et al. [91] investigated the antibacterial effect of the aqueous extract of 12 medicinal plants of Apiaceae family including $F$. vulgare. An aqueous extract of the aerial part of $F$. vulgare inhibited the growth of Agrobacterium radiobacter pv. tumefaciens, Erwinia carotovora, Pseudomonas fluorescens, and Pseudomonas glycinea (Table 9). An aqueous extract of seed sample inhibited the growth of Enterococcus faecalis, Staphylococcus aureus, Escherichia coli, Klebsiella pneumonia, Pseudomona aeruginosa, Salmonella typhi, Salmonella typhimurium, Shigella flexneri, and Bacillus cereus with 1322 , 22-24, 14-24, 20-21, 21-24, 11-12, 14-18, 17-18, and 24$26 \mathrm{~mm}$ zone of inhibition, respectively $[3,4]$. Gulfraz et al. [92] investigated the antibacterial effect of the essential oil as well as ethanolic and methanolic fruit extracts of F. vulgare against Bacillus cereus, Bacillus megaterium, Bacillus pumilus,
Bacillus subtilis, Escherichia coli, Klebsiella pneumonia, Micrococcus luteus, Pseudomonas putida, Pseudomonas syringae, and Candida albicans. According to the results reported by Gulfraz et al. [92], essential oil of F. vulage had significant antimicrobial activities against some of microorganisms as compared to the methanolic and ethanolic extracts. The diameters of growth inhibition zone ranged from 14 to $31 \mathrm{~mm}$ (including the diameter of the disc $6 \mathrm{~mm}$ ) with the highest inhibition zone values observed against Bacillus megaterium $(31 \mathrm{~mm})$ and Bacillus subtilis $(29 \mathrm{~mm})$. Roby et al. [23] investigated antimicrobial effect of the methanol, ethanol, diethyl ether, and hexane extracts of seed of $F$. vulgare against two species of Gram negative bacteria (Escherichia coli and Salmonella typhi), two species of Gram positive bacteria (Bacillus cereus and Staphylococcus aureus), one species of yeast (Candida albicans), and one species of mold (Aspergillus flavus). The methanolic extract showed more effective antimicrobial activity than the other extracts. The results from the disc diffusion method, followed by measurement of minimum inhibitory concentration (MIC), indicated that Bacillus cereus and Aspergillus flavus were the most sensitive microorganisms tested, showing the largest inhibition zones and the lowest MIC values. Least activity was exhibited against Escherichia coli, with the smallest inhibition zones and the highest MIC value [23]. Shrivastava and Bhargava [93] investigated the antibacterial effect of the crude, chloroform, and methanol extract of leaves and flowers of $F$. vulgare along with Raphanus sativus and Brassica nigrum against Escherichia coli and Staphylococcus aureus. Methanol extract of flower of F. vulgare showed significant activity against Escherichia coli, whereas crude and chloroform extracts failed to exhibit antimicrobial activity against Staphylococcus aureus (Table 9). Among different tested bacterial strains, the 
TABLE 6: Volatile compounds present in essential oil of Foeniculum vulgare.

\begin{tabular}{|c|c|}
\hline Sr. number & Compounds \\
\hline 1 & $\alpha$-Thujene \\
\hline 2 & 1,8-Cineol \\
\hline 3 & $\beta$-Ocimene \\
\hline 4 & Linalool \\
\hline 5 & Germacrene D \\
\hline 6 & Anisketone \\
\hline 7 & Apiol \\
\hline 8 & $n$-Hexadecanoic acid \\
\hline 9 & Cubebene \\
\hline 10 & Benzene-1-methyl-4-(1-methylethyl)-p-cymene \\
\hline 11 & 1,3,6-Octatriene, 3,7-dimethyl-, (E)-3-carene \\
\hline 12 & 2-Heptene \\
\hline 13 & 3-Methyl-butanal \\
\hline 14 & $\beta$-Pinene \\
\hline 15 & Camphene \\
\hline 16 & Hexanal \\
\hline 17 & $\alpha$-Pinene \\
\hline 18 & $\beta$-Phellandrene \\
\hline 19 & $\alpha$-Phellanrrene \\
\hline 20 & $\beta$-Myrcene \\
\hline 21 & 4-Carene \\
\hline 22 & 2-Heptanohe \\
\hline 23 & Limonene \\
\hline 24 & 4-Methyl-bicyclo[3.1.0]hex-2-ene \\
\hline 25 & Eucalyptol \\
\hline 26 & $\alpha$-Pinene \\
\hline 27 & $\gamma$-Terpinene \\
\hline 28 & 7-Dimethyl-1,3,7-octriene \\
\hline 29 & 2,4-Dimethyl-benzenamine \\
\hline 30 & 3-Carene \\
\hline 31 & Cathine \\
\hline 32 & 2-Heptanol \\
\hline 33 & 2-Propyn-1-ol \\
\hline 34 & 2,6-Dimethyl-2,4,6-octatriene \\
\hline 35 & Fenchone \\
\hline 36 & 1-Methyl-4-(1-methylethyl)-benzene \\
\hline 37 & cis-Limonene oxide \\
\hline 38 & trans-Limonene oxide \\
\hline 39 & 6-Methylene-bicyclo[3.1.0]hexane \\
\hline 40 & Sabinene hydrate \\
\hline 41 & Fenchyl acetate \\
\hline 42 & Camphor \\
\hline 43 & Benzaldehyde \\
\hline 44 & 1,3-Butanediol \\
\hline 45 & Dicyclopropyl carbinol \\
\hline 46 & Fenchol \\
\hline 47 & 1-Octanol \\
\hline 48 & 5-Methyl-2-heptanol \\
\hline
\end{tabular}

TABLE 6: Continued.

\begin{tabular}{|c|c|}
\hline Sr. number & Compounds \\
\hline 49 & Tetradecyl-oxirane \\
\hline 50 & Estragole \\
\hline 51 & Trans- $p$-2,8-menthadien-1-ol \\
\hline 52 & $\beta$-Terpinol \\
\hline 53 & cis-p-2,8-Menthadien \\
\hline 54 & 4-Methyl-1-(methylethyl)-3-cyclohexen \\
\hline 55 & 2-Methyl-5-(1-methylethyl)-2-cyclohexen-1-one \\
\hline 56 & Phenylmethyl-formic ester \\
\hline 57 & 2,3-Cyclohexen-1-methanol \\
\hline 58 & Epi-bicyclosesquiphellardrene \\
\hline 59 & cis-p-Menth-2,8-dienol \\
\hline 60 & 1,4-Dimethoxy-benzene \\
\hline 61 & 1-Methoxy-4-(1-propenyl)-benzene \\
\hline 62 & 1,2,4a,5,8,8a-Hexadehyde-naphthalene \\
\hline 63 & 4-Methyl-bicyclo[3.1.1]hept-3-en-2-ol \\
\hline 64 & trans-Anethole 73.2073 .2766 .71 \\
\hline 65 & Allantoic acid \\
\hline 66 & 2-Methyl-5-(1-methylethyl)-phenol \\
\hline 67 & Mannoheptulose \\
\hline 68 & 2-Methyl-5-(1-methylethyl)-2-cyclohexen-1-ol \\
\hline 69 & 1-Undecanol \\
\hline 70 & Benzothiazole \\
\hline 71 & E-Pinane \\
\hline 72 & 2-Cyclohexen-1-ol \\
\hline 73 & 2-Methyl-bezenemethanol \\
\hline 74 & 4-Methoxy-benzaldehyde \\
\hline 75 & 1,6-Hexanediol \\
\hline 76 & 2-Methoxycyclohexanone \\
\hline 77 & $\beta$-Elemenone \\
\hline 78 & Mephenesin \\
\hline 79 & $4^{\prime}$-Methoxy-acetophenone \\
\hline 80 & 2-Methyl-3-methylethyl-butanoic acid \\
\hline 81 & Folic acid \\
\hline 82 & 1-(Methoxyphenyl)-2-propanone \\
\hline 83 & 1-Methyl-3-(1-methylethyl)-benzene \\
\hline 84 & 4-Fluorohistamine \\
\hline 85 & 1,2-Dimethoxy-4-(1-propenyl)-benzene \\
\hline 86 & (E)-2-Hydroxy-4-cyano-stilbene \\
\hline 87 & 1-(3-Methoxyphenyl)-1-propanone \\
\hline
\end{tabular}

methanolic fruit extract of $F$. vulgare inhibited the growth of Staphylococcus aureus and Bacillus pumilus with 11.27 and $12.67 \mathrm{~mm}$ zone of inhibition, respectively [7].

Several studies indicating the antifungal effect of $F$. vulgare along with antibacterial effect are also reported in the literature. Martins et al. [94] investigated the antibacterial and antifungal effects of three essential oils of Portuguese plants, namely, Foeniculum vulgare, Mentha spicata, and Rosmarinus officinalis against Staphylococcus aureus, Escherichia coli, Klebsiella pneumonia, Pseudomona aeruginosa, Staphylococcus epidermidis, Candida albicans, and phytopathogenic 
TABLE 7: Biological activities of some phytoconstituents reported in different parts of Foeniculum vulgare.

\begin{tabular}{|c|c|c|c|c|}
\hline Sr. number & Biological activities & Part used $^{\mathrm{a}}$ & Phytochemicals & Reference \\
\hline 1 & Oestrogenic & SDEO & $\begin{array}{l}\text { Dianethole, } \\
\text { photoanethole }\end{array}$ & {$[71]$} \\
\hline 2 & Hepatoprotective & SDEO & $\begin{array}{c}\beta \text {-Myrcene, } \\
\text { Limonene }\end{array}$ & [9] \\
\hline 3 & Antithrombotic & SDEO & trans-Anethole & {$[55]$} \\
\hline 4 & $\begin{array}{l}\text { Human liver cytochrome } \\
\text { P450-3A4 inhibitory }\end{array}$ & SD & 5-Methoxypsoralen & {$[83]$} \\
\hline \multirow[t]{2}{*}{5} & \multirow[t]{2}{*}{ Antiradical scavenging } & FW & $\begin{array}{l}\text { 3-Caffeoylquinic acid, } \\
\text { quercetin-3-O-galactoside, } \\
\text { kaempferol-3-O-glucoside, } \\
\text { kaempferol-3-O-rutinoside, } \\
\text { rosmarinic acid }\end{array}$ & {$[67]$} \\
\hline & & $\mathrm{AP}$ & $\begin{array}{c}\text { 3,4-Dihydroxyphenethyl- } \\
\text { alchohol-6-O-caffeoyl- } \beta \text {-D- } \\
\text { glucopyranoside, } \\
3^{\prime}, 8^{\prime} \text {-binaringenin }\end{array}$ & {$[70]$} \\
\hline 6 & Antioxidant & FT & cis-Miyabenol C & {$[139]$} \\
\hline 7 & Anticancer & SDEO & Anethole & {$[110]$} \\
\hline 8 & Antibacterial & ST & $\begin{array}{l}\text { Dillapiol, } \\
\text { psoralen, } \\
\text { bergapten, } \\
\text { scopoletin, } \\
\text { imperatorin, } \\
\text { dillapional, }\end{array}$ & {$[100]$} \\
\hline 9 & Antimycobacterial & ST, LF & $\begin{array}{l}\text { 2,4-Undecadienal, } \\
\text { linoleic acid, } \\
\text { oleic acid, } \\
\text { 1,3-benzenediol, } \\
\text { undecanal }\end{array}$ & {$[63]$} \\
\hline 10 & Repellent & FT & $\begin{array}{c}\text { (z)-9-Octadecanoic acid, } \\
\text { fenchone }\end{array}$ & {$[117]$} \\
\hline 11 & Acaricidal & SDEO & para-Anisaldehyde & [118] \\
\hline 12 & Insecticidal & SDEO & $\begin{array}{l}\text { 1,8-Cineole, } \\
\text { terpineol }\end{array}$ & [120] \\
\hline
\end{tabular}

aP: aerial part, FT: fruit, LF: leaf, SD: seed, SDEO: seed essential oil, ST: stem, and FW: fennel waste.

molds, Aspergillus niger and Fusarium oxysporum. Essential oil of $F$. vulgare showed significant antifungal activity against the food spoilage fungi Aspergillus niger and Fusarium oxysporum and may have important applications as food additives. The MIC values of $F$. vulgare essential oil were $250 \mu \mathrm{g} / \mathrm{mL}$ for Fusarium oxysporum and $750 \mu \mathrm{g} / \mathrm{mL}$ for Aspergillus niger [94]. The oils extracted from F. vulgare exhibit varying levels of antifungal effects on the experimental mycelial growth of Alternaria alternata, Fusarium oxysporum, and Rhizoctonia solani [95]. Essential oil of F. vulgare showed appreciable antifungal activity against strains of pathogenic fungi, namely, Aspergillus niger, Fusarium solani, and Rhizopus solani [96]. Dichloromethane extracts and essential oils from $F$. vulgare showed antifungal activity against Candida albicans. It could be a potential candidate for a new antifungal agent for candidiasis and other fungal diseases [97]. In an in vitro study, aqueous and alcoholic seed extracts of $F$. vulgare exhibited inhibitory effect against Alternaria alternata, Mucor rouxii, and Aspergillus flavus [98]. Interestingly, aqueous seed extract of $F$. vulgare showed strongest antifungal activity as compared to reference fungicidal agent, that is, griseofulvin [99].

All of the above mentioned studies were carried out on the crude extracts and it is difficult to pinpoint the active antimicrobial metabolite. A phenylpropanoid derivative called dillapional, characterized from $F$. vulgare stem, was found to be an antimicrobial constituent with MIC values of 125, 250, and 125 against Bacillus subtilis, Aspergillus niger, and Cladosporium cladosporioides, respectively. A coumarin derivative, scopoletin, was also isolated as a marginally antimicrobial agent [100]. The characterization of seven different types of oxygenated monoterpenes, from methylene 


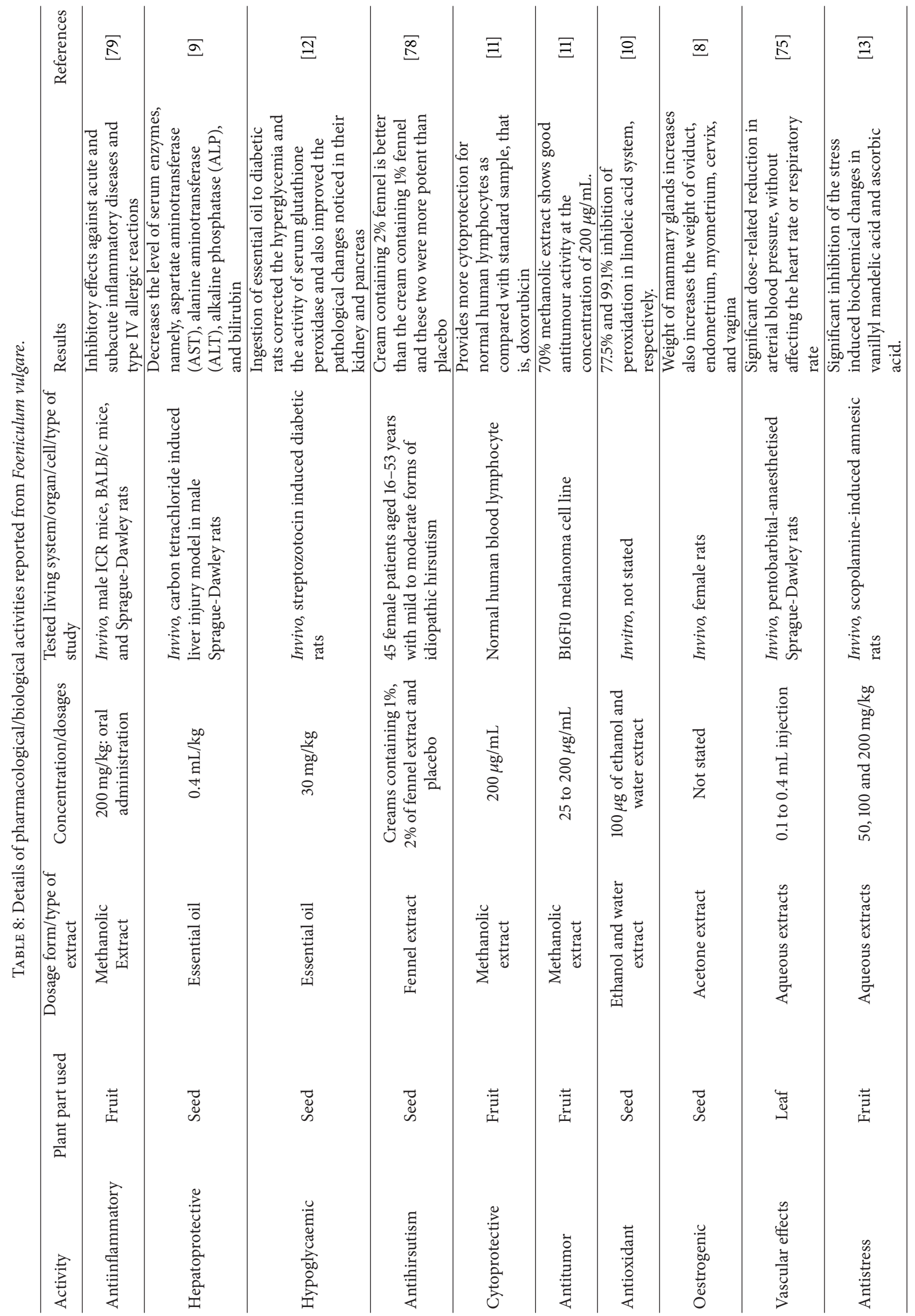




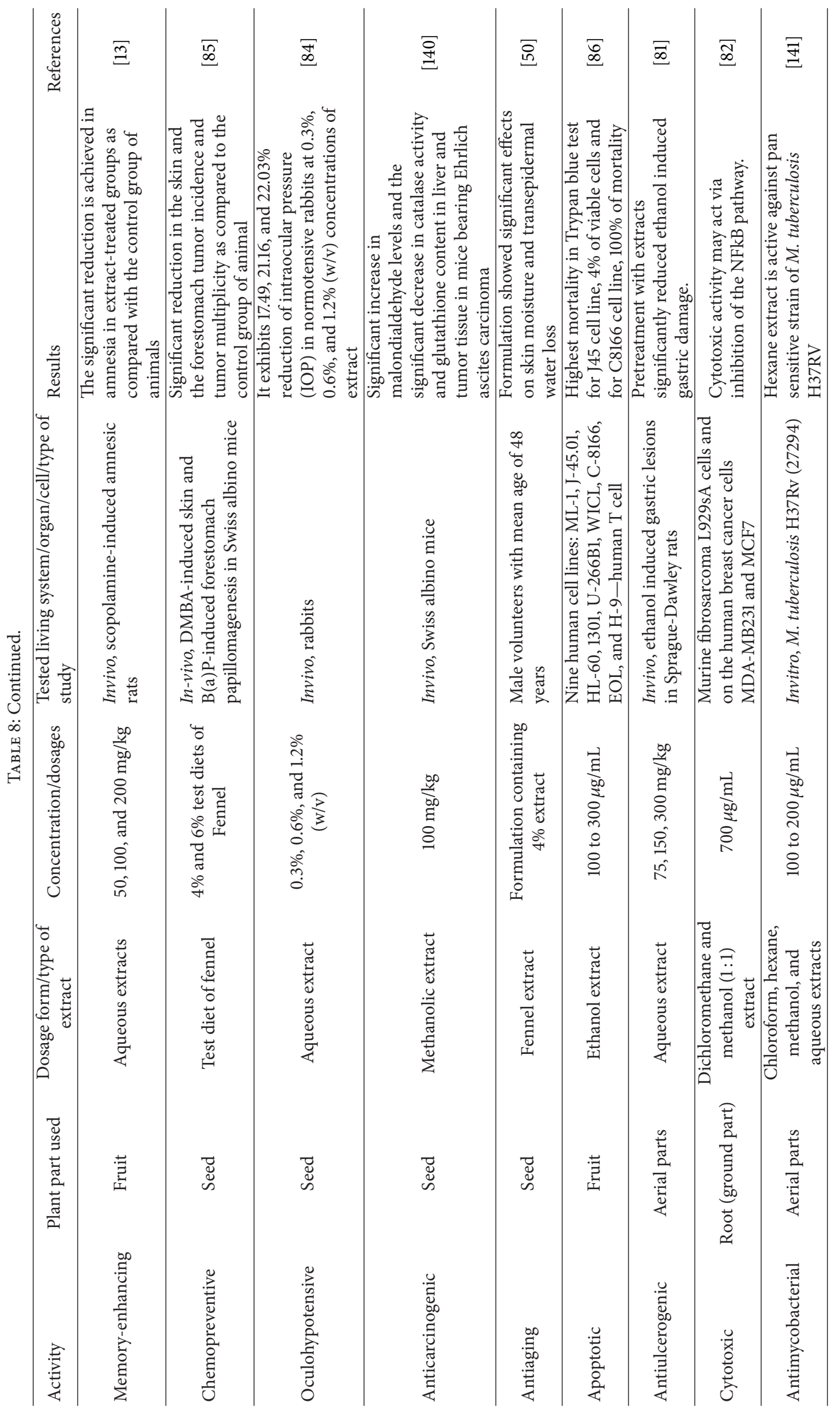




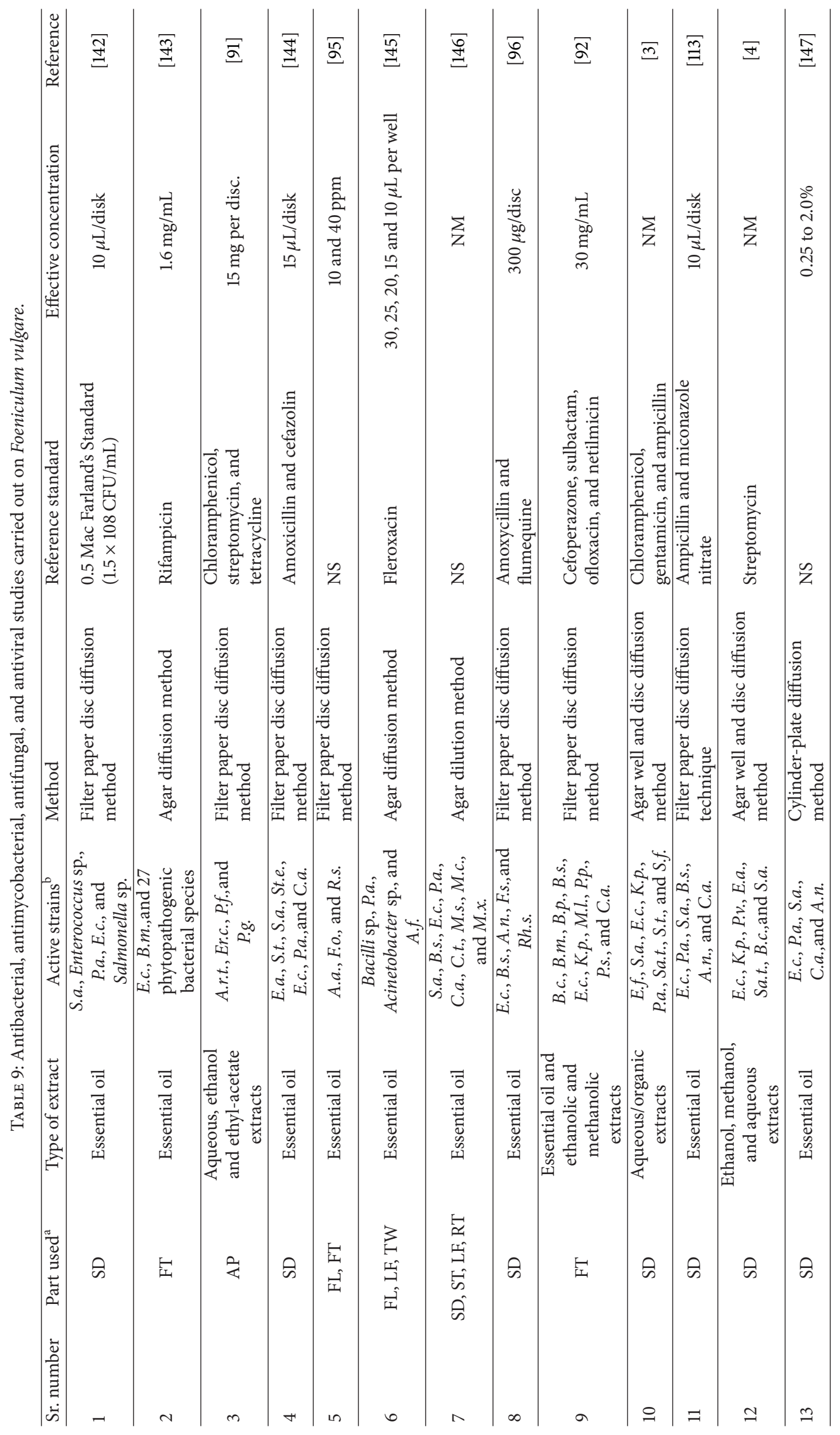




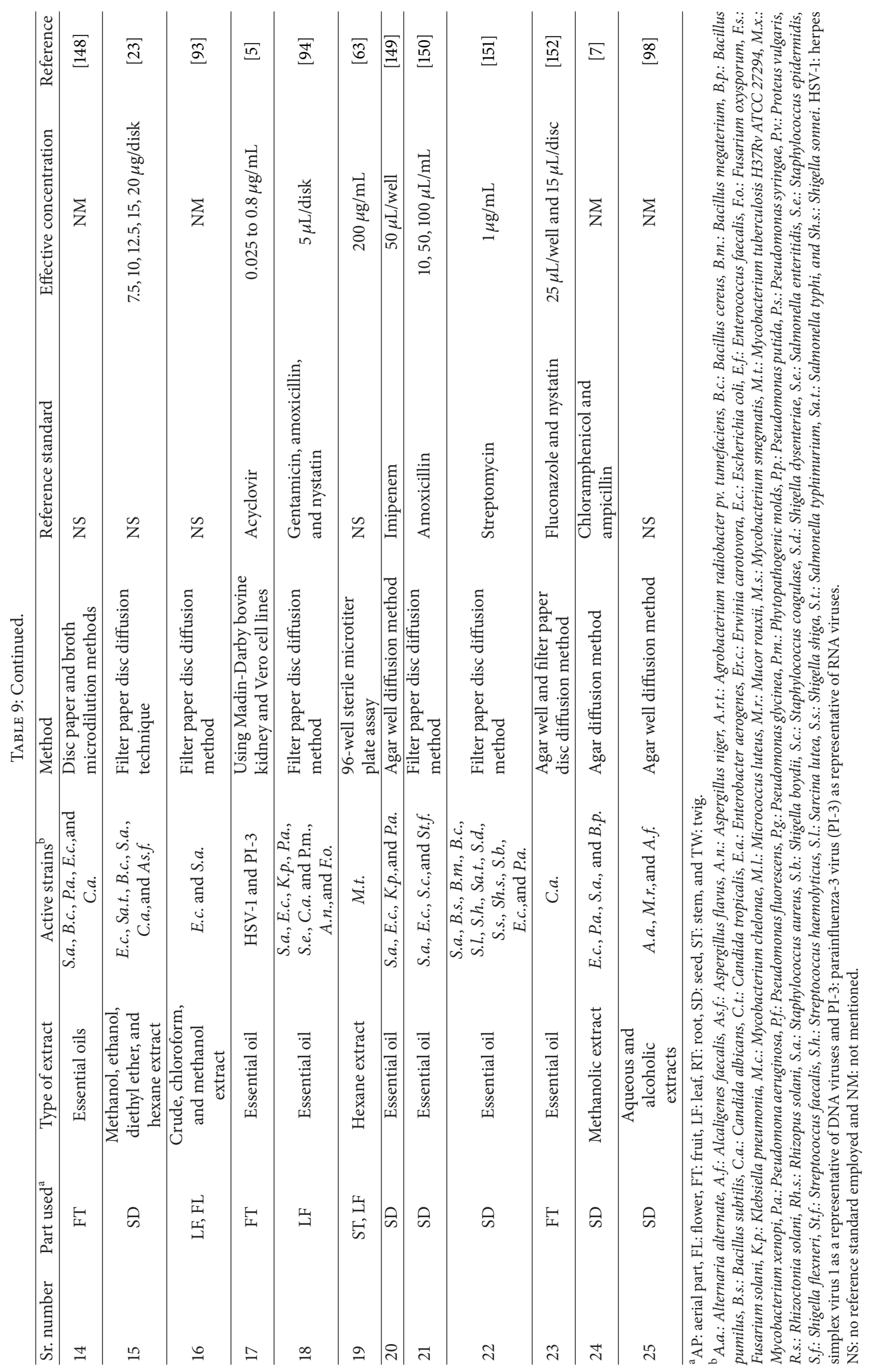


chloride crude extract of F. vulgare [101], suggested that the crude extract containing monoterpenes could be a new medicinal resource for antibacterial agents.

A total of 78 compounds were identified from the active antimycobacterial fraction of $F$. vulgare with the help of gas chromatography-mass spectra (GC-MS). Out of these, twenty compounds were tested against one sensitive and three MDR strains of Mycobacterium tuberculosis using the Alamar Blue microassay. Compounds that showed some degree of antimycobacterial activity against all strains tested were the following: linoleic acid (MIC $100 \mu \mathrm{g} / \mathrm{mL}$ ), oleic acid (MIC $100 \mu \mathrm{g} / \mathrm{mL}$ ), 1,3-benzenediol (MIC 100$200 \mu \mathrm{g} / \mathrm{mL}$ ), undecanal (MIC 50-200 $\mu \mathrm{g} / \mathrm{mL}$ ), and 2,4undecadienal (MIC $25-50 \mu \mathrm{g} / \mathrm{mL}$ ). 2,4-Undecadienal was the most active compound against multidrug resistant $M$. tuberculosis species. Thus, the dietary intake of $F$. vulgare may lower the risk of $M$. tuberculosis infection [63].

Orhan et al. [5] studied the antiviral activity of the essential oil of fruit sample of $F$. vulgare along with 12 other Turkish medicinal plants against the DNA virus Herpes simplex type-1 (HSV-1) and the RNA virus parainfluenza type-3 (PI-3). Most of the oils and compounds displayed strong antiviral effects against $H S V-1$, ranging between 0.8 and $0.025 \mu \mathrm{g} / \mathrm{mL}$. However, the samples tested were less effective against PI-3, with results ranging between 1.6 and $0.2 \mu \mathrm{g} / \mathrm{mL}$. Only the essential oils of Anethum graveolens, Foeniculum vulgare (fully mature), Mentha piperita, Mentha spicata, Ocimum minutiflorum, Ocimum vulgaris, and Satureja cuneifolia inhibited this virus significantly.

All these literature findings validated the traditional uses of Foeniculum vulgare in infectious disorders like abdominal pains, antiemetic, arthritis, conjunctivitis, constipation, depurative, diarrhea, dieresis, fever, flatulence, gastralgia, gastritis, insomnia, irritable colon, mouth ulcer, stomachache, respiratory disorders, skin diseases, and so forth. There is always a need for new antimicrobial agents due to rapid development of resistance. Bioactive metabolites of $F$. vulgare may be a potential source for new antimicrobial agents.

4.2. Anti-Inflammatory Activity. Oral administration of methanol extract of $F$. vulgare fruit to rat and mice exhibited inhibitory effects against acute and subacute inflammatory diseases. The anti-inflammatory activity of methanol extract was evaluated by using three screening protocols, namely, carrageenan-induced paw edema, arachidonic acid-induced ear edema, and formaldehyde-induced arthritis. These are widely used for testing nonsteroidal anti-inflammatory drugs. For acute inflammation, methanol extract $(200 \mathrm{mg} / \mathrm{kg})$ exhibits significant inhibition of paw edema (69\%) induced by carrageenan injection as compared to the control group of animals. Methanol extract of $F$. vulgare also inhibits ear-edema $(70 \%)$ induced by arachidonic acid in mice. The level of serum transaminase, aspartate aminotransferase (AST), and alanine aminotransferase (ALT) significantly increases in the presence of methanolic extract of $F$. vulgare on inflammation induced by formaldehyde as compared to control group. The assessment of the level of AST and
ALT provides a good and simple tool to measure the antiinflammatory activity of the target compounds [102]. These overall results seem to suggest that $F$. vulgare FME may act on both the cyclooxygenase and lipoxygenase pathways [79].

4.3. Antiallergic Activity. Methanolic extract of F. vulgare fruit showed significant inhibitory effect on DNFB- $(2,4-$ dinitrofluorobenzene-) induced delayed type hypersensitivity after oral administration of $200 \mathrm{mg} / \mathrm{kg}$ once a day for 7 days. The inhibitory effect on immunologically induced swelling suggests the possible immunosuppressive properties of F. vulgare [79].

4.4. Hepatoprotective Activity. Essential oil of F. vulgare seeds revealed a potent hepatoprotective effect against acute hepatotoxicity produced by carbon tetrachloride in rats. Oral administration of $F$. vulgare essential oil decreases the levels of serum aspartate aminotransferase (AST), alanine aminotransferase (ALT), alkaline phosphatase (ALP), and bilirubin as compared to the control group. Ozbek et al. suggest that the constituents ( $d$-limonene and $\beta$-myrcene) of essential oil may have played a key role in the protection of liver from $\mathrm{CCl}_{4}$ toxicity [9].

4.5. Anxiolytic Activity. Anxiety is the unpleasant feeling of fear and concern. When anxiety becomes excessive, it may be considered as an anxiety disorder. Anxiolytic fennel is a drug used for the treatment of anxiety and its related psychological and physical symptoms. Naga Kishore et al. [89] investigated the anxiolytic activity of ethanolic extract of F. vulgare fruit with the help of elevated plus maze, rota rod, open field test, and whole board models. The 100 to $200 \mathrm{mg}$ dose of extract per $\mathrm{kg}$ of body weight of animal revealed significant activity when compared to reference anxiolytic drug called diazepam $(1 \mathrm{mg} / \mathrm{kg})$. Thus, fennel extract may possess anxiolytic activity supporting its traditional claim about anxiolytic activity reported in 19th edition of Pharmacology and Pharmacotherapeutics by Sathodkar, Bhandarkar and Rege.

4.6. Antistress Activity. Drug and food of natural origin play a significant role in public healthcare systems and are being investigated as remedies for a number of stress-related disorders [103]. The whole plant extract of $F$. vulgare exhibited notable antistress effect against stress induced by forceful swimming of test animals. The key parameters, that is, urinary levels of vanillyl mandelic acid (VMA) and ascorbic acid in rats were used to evaluate antistress activity. The plant extract (50, 100 and $200 \mathrm{mg} / \mathrm{kg}$ body weight) showed a significant improvement in urinary levels of VMA $(P<$ $0.001)$, and ascorbic acid excretion levels $(P<0.001)$, in test animals when compared to the normal basal levels in control group of animals. Thus, the extract of entire plant of $F$. vulgare acts as an antistress agent [13].

4.7. Memory-Enhancing Property. There are a number of plants, whose consumption is believed to enhance memory 
and intelligence. These were usually given to children as part of their food. F. vulgaris an ayurvedic rasayana (mixture) possessing multiple neuropharmacological activities. The antidepressant activity of fennel has been well documented in ethnomedicine. The whole plants extract (50, 100 and $200 \mathrm{mg} / \mathrm{kg}$ ) of F. vulgare exhibited memory-enhancing effect against scopolamine-induced amnesic rats. This experiment was evaluated by conditioned avoidance response (CAR) technique. The CAR of rats administered with the extract increased gradually to $95 \%$ over 7 to 12 days. The acquisition (time to achieve 95\% CAR) for rats administered with the extract was dose- and time-dependent compared to control group, which took 12 days for acquisition. The percent avoidance was always higher in the extract-treated groups as compared to control group. Animals receiving $200 \mathrm{mg} / \mathrm{kg}$ body weight of the extract took ten days, while groups treated with 100 and $50 \mathrm{mg} / \mathrm{kg}$ doses of the extract required eleven and twelve days, respectively, to reach the point of acquisition. Administration of scopolamine produced amnesia as seen from reduction in the observed CAR. Amnesia was greater in the control group than in extract-treated groups. However, continued treatment with $F$. vulgare produced better retention and recovery in a dose-dependent manner than the vehicle-treated animals. Recovery from scopolamineinduced amnesia in the extract-treated groups took 3-5 days when compared to normal (control) group which took over 6 days. This overall progress suggests that $F$. vulgare extract possesses memory-enhancing property [13].

4.8. Nootropic Activity. Alzheimer's disease is a neurodegenerative disorder associated with a decline in cognitive abilities. Dementia is one of the age-related mental problems and a characteristic symptom of Alzheimer's disease. There is some evidence in favor of use of $F$. vulgare for the treatment of cognitive disorders like dementia and Alzheimer's disease. Methanol extract of the whole plant of F. vulgare administered for eight successive days ameliorated the amnesic effect of scopolamine and aging-induced memory deficits in mice. This extract increased step-down latency and acetylcholinesterase inhibition in mice significantly. Thus, $F$. vulgare may be employed in treatment of cognitive disorders such as dementia and Alzheimer's disease as a nootropic and anticholinesterase agent [80].

4.9. Antihirsutism Activity. Idiopathic hirsutism is defined as the occurrence of excessive male pattern hair growth in women who have a normal ovulatory menstrual cycle and normal levels of serum androgens. It may be a disorder of peripheral androgen metabolism. Traditionally, Foeniculum vulgare has been used as an estrogenic agent. It has been reputed to increase milk secretion, promote menstruation, facilitate birth, and increase libido. On considering above aspect, Javidnia and his research team evaluated the antihirsutism activity of ethanolic extract of $F$. vulgare seed against idiopathic hirsutism by preparing cream containing 1 and $2 \%$ of fennel extract. The efficacy of treatment with the cream containing $2 \%$ fennel is better than the cream containing $1 \%$ fennel and these two were more potent than placebo (control group). The mean values of hair diameter reduction were $7.8 \%, 18.3 \%$, and $-0.5 \%$ for patients receiving the creams containing $1 \%, 2 \%$, and $0 \%$ (placebo), respectively [78].

4.10. Estrogenic Properties. Since the discovery of the estrus inducing effects of some plant products in 1926, considerable effort has been devoted towards the characterization of phytoestrogens, including flavonoids, isoflavonoids, chalcones, coumestans, stilbenes, lignans, saponins, and essential oils [16]. F. vulgare has estrogen-like activity. In male rats, total concentration of protein was found to be significantly decreased in testes and in vasa deferentia whereas increased in seminal vesicles and in prostate gland. On the other hand, simultaneous decrease in the activities of acid and alkaline phosphatase in all these regions (except that alkaline phosphatase was unchanged in vasa), due to the oral administration of acetone extract of $F$. vulgare fruit, was observed. In female rats oral administration of the extract for 10 days led to vaginal cornification and oestrus cycle [8]. Total concentration of nucleic acids and protein as well as the organ weights increased in both the tissues, namely, mammary glands and oviducts, due to the oral administration of acetone extract $(50,150$, and $250 \mu \mathrm{g} / 100 \mathrm{~g}$ body wt) of $F$. vulgare seeds [104]. Fennel oil was reported to exhibit estrogenic activity, promote menstruation and alleviate the symptoms of female climacteric, and increase libido [71]. Administration of fennel oil ( 25 and $50 \mu \mathrm{g} / \mathrm{mL}$ final concentration in the organ bath) failed to exhibit any remarkable effect in uterine contraction. While 10,20 and $40 \mu \mathrm{g} / \mathrm{ml}$ concentration of fennel oil revealed significant inhibitory effect against prostaglandin E2. Fennel oil significantly reduces the frequency of uterine contraction induced by prostaglandin E2. Thus, the extracts of F. vulgare have strong estrogenic activity [76].

4.11. Galactogenic Activity. Foeniculum vulgare has been used for millennia to increase milk secretion [105]. Thus, F. vulgare belongs to galactagogue substance. Structural similarity of its main constituent, anethole, to dopamine seems to be responsible for galactogenic activity. Dopamine acts to inhibit the secretion of the milk-producing hormone, prolactin. Anethole might influence milk secretion by competing with dopamine at the appropriate receptor sites, thereby inhibiting the antisecretory action of dopamine on prolactin [71]. It was reported that anol (demethylated anethole) causes growth of the lobule-alveolar system in the mammary glands of immature female rabbits and induces menstruation in mice and other experimental animals. Anol also gave positive results in the Jadassohn nipple test, a test which involves the measurement of changes induced in the nipples of guinea pigs subjected to the cutaneous application of sex hormones. However, further research suggests that the actual pharmacologically active agents responsible for galactogenic activity are polymers of anethole, such as dianethole and photoanethole, rather than anol or anethole itself $[20,76]$.

4.12. Expectorant Activity. F. vulgare seeds stimulate the ciliary motility of the respiratory apparatus and enhance the 
external transport of extraneous corpuscles. This action suggests a use for fennel in treating bronchial and bronchopulmonary afflictions and in particularly polluted environments [106]. The volatile oil of $F$. vulgare stimulates the contraction of the smooth muscles of the trachea, an action that could facilitate the expectoration of mucus, bacteria, and other corpuscles extraneous to the respiratory tracts [74].

4.13. Anticolitic Activity. Essential oil of fennel regulates the motility of smooth muscles of the intestine, while, at the same time, reducing intestinal gas. Alone, or combined with other plant medicinals, Foeniculum vulgare is indicated in the treatment of spastic gastrointestinal disturbances, in some forms of chronic colitis (which resist other treatments), in dyspepsias from gastrointestinal atony, in dyspepsias with the sensation of heaviness in the stomach, and so forth.The addition of fennel to preparations containing anthraquinonic components reduces the occurrence of abdominal pain often associated with this type of laxative [73].

4.14. Antinociceptive Activity. Antinociceptive means any substance that inhibits nociception which is a physiological process underlying the sensation of pain. Briefly, it reduces the sensitivity to painful stimuli. The various extracts of aerial part of F. vulgare, namely, hexane, methylene chloride, ethyl acetate, and methanolic extract showed remarkable antinociceptive activity against acetic acid induced writhing in mice [68]. The methanolic extract of the aerial parts of F. vulgare exhibited the highest antinociceptive activity at a dose level of $2000 \mathrm{mg} / \mathrm{kg}$, while the activity exhibited by the ethyl acetate extract was at dose level $800 \mathrm{mg} / \mathrm{kg}$. On the other hand, $n$-hexane extract $(700 \mathrm{mg} / \mathrm{kg}$ ) and methylene chloride extract $(500 \mathrm{mg} / \mathrm{kg})$ exhibited similar antinociceptive activities, being less than peripheral antinociceptive reference drug (acetyl salicylic acid) [68].

4.15. Diuretic Activity. A diuretic is any substance that promotes the production of urine. Briefly, it is an agent that promotes diuresis. Diuretics work by promoting the expulsion of urine (measured as the urine volume [UV] excreted) and urinary sodium (UNa) from the body and this helps reduce the volume of blood circulating through the cardiovascular system. Caceres et al. [107] performed a study in conscious animals and administered a powdered extract of the whole plant (F. vulgare) which had no effect on UV or UNa. The ethanolic extract of $F$. vulgare fruit revealed excellent diuretic activity and proves the earlier folk claim of F. vulgare, which was reported in the United State of America (Table 5). The fruit extract showed, statistically, a highly significant diuretic effect. F. vulgare induced diuresis (500 $\mathrm{mg} / \mathrm{kg}$ dose) was comparable to that of reference diuretic agent urea $(960 \mathrm{mg} / \mathrm{kg}$ dose) in mice with a urine output that was almost double that of the control group. The diuresis was not associated with changes in sodium and/or potassium excretion [108]. In another part of the study, the authors showed that Foeniculum vulgare had little effect on the noradrenalin contractile responses of aortic rings, thus suggesting that it worked mainly as a diuretic and natriuretic with little effect on arterial vascular tone [77].

4.16. Cardiovascular Activity. An aqueous extract of F. vulgare leaves possesses potential cardiovascular action. This effect was investigated using pentobarbital-anaesthetised male albino Sprague-Dawley rats [75]. An intravenous administration of the lyophilized boiled water extract of leaves produced a significant dose-related reduction in arterial blood pressure, without affecting the heart rate or respiratory rate. On the other hand the nonboiled aqueous extract showed very little hypotensive activity. The hypotensive effect of the boiling water extract appeared not to be mediated via adrenergic, muscarinic, ganglionic, or serotonergic receptors; however, histamine antagonists inhibited the hypotensive effect in a dose-related manner [75].

4.17. Oculohypotensive Activity. The aqueous seed extract of F. vulgare demonstrated significant oculohypotensive activity using water loading and steroid induced glaucoma model. This extract exhibited $17.49,21.16$, and $22.03 \%$ reduction of intraocular pressure in normotensive rabbits at $0.3 \%, 0.6 \%$, and $1.2 \%(\mathrm{w} / \mathrm{v})$ concentrations, respectively. A maximum mean difference of $31.20 \%$ was observed between vehicles treated and extracts treated eyes in water loading experimental animal model while a maximum mean intraocular pressure lowering of $31.29 \%$ was observed in steroid induced model of glaucoma. Thus, the aqueous extract of $F$. vulgare revealed oculohypotensive activity, which was found to be as good as that of reference standard antiglaucoma drugs called timolol [84].

4.18. Antithrombotic Activity. Tognolini et al. [55] provided evidence of potent inhibitory activity of essential oil of $F . v u l$ gare against platelet aggregation induced by ADP, arachidonic acid, and collagen in guinea pig plasma. Similar findings were reported by Yoshioka and Tamada [109] for aggregation of rabbit platelets by an aromatic factor of fennel oil. The essential oil and anethole (a constituent of oil) of F. vulgare, tested in vitro in rat aorta with or without endothelium, displayed comparable NO-independent vasorelaxant activity at antiplatelet concentrations. It supports the safety of $F$. vulgare, that is, free from cytotoxic effects. Anethole and $F$. vulgare did not cause cytotoxicity when incubated for $30 \mathrm{~min}$ upto $300 \mu \mathrm{g} / \mathrm{mL}$ in platelet viability test. This concentration was largely compatible with those adopted in the functional in vitro tests. In vivo, both F. vulgare essential oil and anethole orally administered in a subacute treatment to mice (30 mg/kg/day for 5 days) showed significant antithrombotic activity preventing the paralysis induced by collagenepinephrine intravenous injection $(70 \%$ and $83 \%$ protection, resp.). Thus, essential oil and its main component anethole of F. vulgare showed a safe antithrombotic activity in guinea pig plasma that seems due to their broad spectrum antiplatelet activity, clot destabilizing effect, and vasorelaxant action [55].

4.19. Antimutagenic Effect. Essential oil of F. vulgare revealed noteworthy protective effects against genotoxicity in mice 
induced by cyclophosphamide. Genotoxicity and cytotoxicity were assessed by using mice bone marrow chromosomal aberration, micronucleus, and sperm abnormality assays, respectively. Oral administration of essential oil (1 and $2 \mathrm{~mL} / \mathrm{kg}$ ) significantly inhibited the frequencies of aberrant metaphases, chromosomal aberrations, micronuclei formation, and cytotoxicity in mouse bone marrow cells induced by cyclophosphamide and also produced a significant reduction of abnormal sperm and antagonized the reduction of cyclophosphamide induced superoxide dismutase, catalase, and glutathione activities and inhibited increased malondialdehyde content in the liver. Additionally, F. vulgare inhibits the oxidative stress induced by cyclophosphamide [90].

4.20. Gastrointestinal Effect. The aqueous extract of $F$. vulgare showed remarkable antiulcerogenic effect against ethanolinduced gastric lesions in rats. It was found that pretreatment with aqueous extract significantly reduced ethanol-induced gastric damage. This effect of aqueous extract was highest and statistically significant in $300 \mathrm{mg} / \mathrm{kg}$ group compared with the control $(P<0.001)$ group of animal. Additionally, aqueous extract of $F$. vulgare significantly reduced the whole blood malondialdehyde levels, while significantly increased nitrite, nitrate, ascorbic acid, retinol, and beta-carotene levels. Thus, aqueous extract of $F$. vulgare fruit had clearly a protective effect against ethanol-induced gastric mucosal lesion in rats [81].

4.21. Chemomodulatory Action. The chemopreventive effect of different doses of test diet of Foeniculum vulgare seeds was examined against 7,12-dimethylbenz(a)anthracene- (DMBA) induced skin papillomagenesis and benzo(a)pyrene[B(a)P-] induced forestomach papillomagenesis, at the periinitiational level in Swiss albino mice. Fennel seeds exhibit a significant reduction in the skin and the fore-stomach tumor incidence and tumor multiplicity as compared to the control group. Further, biochemical assays showed a significant increase in the content/activities of phase I enzymes especially in the case of $6 \%$ test diet. A concomitant increase in the activities of the phase II enzymes was observed with all the doses of test diet under study. A significant enhancement in the activities of antioxidant enzymes was observed especially at $4 \%$ and $6 \%$ test diets of fennel. These findings were indicative of chemopreventive potential of fennel against carcinogenesis. This is the first report showing chemopreventive potential of seeds of fennel against carcinogenesis [85].

4.22. Cytoprotection and Antitumor Activity. Anethole is the principal active component of fennel seeds which has exhibited anticancer activity. Al-Harbi et al. studied the antitumor activity of anethole against Ehrlich ascites carcinoma induced in a tumor model in Swiss albino mice. The study revealed that anethole increased survival time, reduced tumor weight, and reduced the volume and body weight of the Ehrlich ascites tumour-bearing mice. It also produced a significant cytotoxic effect in the Ehrlich ascites tumour cells in the paw, reduced the levels of nucleic acids and malondialdehyde, and increased glutathione concentrations [110]. In vitro cytoprotection activity of methanolic extract of Foeniculum vulgare was evaluated against normal human blood lymphocytes by micronucleus assay and antitumor activity against B16F10 melanoma cell line by Trypan blue exclusion assay for cell viability. Lymphocyte culture treated with $70 \%$ methanolic extract of Foeniculum vulgare showed very less percentage of micronucleus, that is, $0.006 \%$ as compared to standard drug doxorubicin which showed $0.018 \%$ micronucleus. On the other hand $70 \%$ methanolic extract of Foeniculum vulgare has potent antitumor activity at the concentration of $200 \mu \mathrm{g} / \mathrm{mL}$. The results suggest that the Foeniculum vulgare could be considered as a natural resource of antitumor agents as well as cytoprotective to normal cells [11].

4.23. Cytotoxicity. Kaileh and his coworker investigated the cytotoxic effect of organic extracts of 24 selected Palestinian medicinal plant species. The plant selection was based on existing ethnobotanic information and interviews with local healers. The extracts of the plants under investigation were tested for their potential antitumor (cytotoxic) effect on the murine fibrosarcoma L929sA cells and on the human breast cancer cells MDA-MB231 and MCF7. The extract from $F$. vulgare presented an IC (50) value at $24 \mathrm{~h}$ of $700 \pm 28$ and $500 \pm 17 \mu \mathrm{g} / \mathrm{mL}$, on L929sA and MCF7 cells, respectively. The nuclear transcription factor $\mathrm{NFkappaB}$ or $\mathrm{NF} \kappa \mathrm{B}$ regulates the expression of various genes. They further investigated the effect of nine promising plant extracts, withheld from the first cell viability screening on $\mathrm{NF} \kappa \mathrm{B}$ activation. The dichloromethane and methanol $(1: 1)$ extract of aerial part of F. vulgare revealed immunomodulatory $\mathrm{NF} \kappa \mathrm{B}$ activities [82]. Also, Berrington and Lall investigated the in vitro cytotoxicity of acetone extracts of F. vulgare and other eight medicinal plants against a noncancerous African green monkey kidney (Vero) cell line and an adenocarcinoma cervical cancer (HeLa) cell line [87].

4.24. Antipyretic Activity. F. vulgare extract showed antipyretic activity against hyperpyrexia in mice. It was induced by S.C. administration of $2 \mathrm{~mL} / 100 \mathrm{~g}$ of a $20 \%$ aqueous suspension of brewer's yeast. As an antipyretic agent, ethanolic extract of $F$. vulgare fruit showed a moderate antipyretic activity that was statistically significant after 30 and $90 \min (P<0.01)$ [108].

4.25. Hypolipidemic Activity. The aqueous extract of F. vulgare revealed notable hypolipidemic and antiatherogenic activity against Triton WR-1339 induced hyperlipidemia in mice. Aqueous extract causes significant reduction of plasma lipid levels, that is, cholesterol, triglycerides, LDLcholesterol, and apolipoprotein-B decreased by $40 \%, 23 \%$, $61 \%$, and $61 \%$, respectively, and increase in HDL-cholesterol and apolipoprotein A1 by $85 \%$ and 58\%, respectively [88].

4.26. Hypoglycemic Activity. The essential oil of F. vulgare exhibits potential hypoglycemic and antioxidant activity against streptozotocin induced diabetes in rats. Essential oil (30 mg/kg body weight) of $F$. vulgare works in the 
correction of hyperglycemia from $162.5 \pm 3.19 \mathrm{mg} / \mathrm{dL}$ to $81.97 \pm 1.97 \mathrm{mg} / \mathrm{dL}$ with $P<0.05$ and the activity of serum glutathione peroxidase from $59.72 \pm 2.78 \mathrm{U} / \mathrm{g} \mathrm{Hb}$ to $99.60 \pm$ $6.38 \mathrm{U} / \mathrm{g} \mathrm{Hb}$ with $P<0.05$. Also, essential oil of fennel improves the pathological changes noticed in their kidney and pancreas as compared with the control group of animal. This can prove its effect as antidiabetic in folk Medicine. This makes the possibility of its inclusion in antidiabetic drug industry [12].

4.27. Antispasmodic Activity. The antispasmodic activity of 2.5 and $10.0 \mathrm{~mL} / \mathrm{L}$ of alcoholic extract of Foeniculum vulgare along with other Germanic medicinal plants, namely, Melissa officinalis, Rosmarinus officinalis, Mentha piperita, Matricaria chamomilla, Carum carvi, and Citrus aurantium were tested employing the guinea pig ileum and using acetylcholine and histamine as spasmogens. An alcoholic extract of the fruits of Foeniculum vulgare possesses antispasmodic activity, which inhibits the acetylcholine and histamine-induced guinea pig ileal contractions in vitro. An essential oil which is obtained from the fruits of Foeniculum vulgare, $25 \mu \mathrm{g} / \mathrm{mL}$ and $10 \mu \mathrm{g} / \mathrm{mL}$, respectively, inhibited oxytocin and prostaglandin [72].

4.28. Apoptotic Activity. The apoptotic activities of ethanol extracts from fruits of seven species of Apiaceae family, namely, Eryngium planum, Archangelica officinalis, Pastinaca sativa, Heracleum sibiricum, Carum carvi, Foeniculum vulgare, and Levisticum officinale against ML-1-human acute myeloblastic leukaemia, J-45.01-human acute T cell leukaemia, EOL-human eosinophilic leukaemia, HL60-human Caucasian promyelocytic leukaemia, 1301human $\mathrm{T}$ cell leukaemia lymphoblast, C-8166-human $\mathrm{T}$ cell leukaemia, U-266B1-human myeloma, WICL-human Caucasian normal B cell, and H-9-human $\mathrm{T}$ cell were investigated with the help of Trypan blue assay and Annexin $\mathrm{V}$ fluos assay [86]. The ethanol extract from fruit F. vulgare showed the highest mortality in Trypan blue test for J45 cell line-4\% of viable cells and for C8166 cell line-100\% of mortality. However the cells of other lines showed the highest viability: HL60-60\%, EOL-48\%, and ML-1-42\%. The normal cell line H9 and WICL showed 35\% and 25\% of viable cells, respectively. C8166 cell line and J-45 cell line showed the highest level of the apoptotic cells detected by Annexin V method-100\% and 93\%, respectively. However the cells of two lines HL60 and EOL-1 showed the lower levels of apoptotic cells-52\% and $60 \%$, respectively. High percentage of apoptotic cells was observed in H9 and WICL$76 \%$ and $93 \%$, respectively [86].

4.29. Human Liver Cytochrome P450 3A4 Inhibitory Activity. Thirteen compounds isolated from the methanolic extract of fennel have been found to possess human liver cytochrome P450 3A4 inhibitory activity. Among these compounds 5methoxypsoralen (5-MoP) showed the strongest inhibition with an IC50 value of $18.3 \mu \mathrm{m}$ and with a mixed type of inhibition [83].
4.30. Antiaging Effects. Rasul and his coworker developed a base and formulation containing $4 \%$ concentrated seed extract of F. vulgare. This formulation shows notable antiaging effect with supporting experimental data related to skin moisture and transepidermal water loss (TEWL). The base was insignificant, while the formulation showed significant effects on skin moisture and TEWL. The texture parameter energy showed a significant increase proving that the formulation possesses potential antiaging effects [50].

4.31. Bronchodilatory Effect. Ethanol extract and essential oil from $F$. vulgare exhibited bronchodilatory activity on contracted tracheal chains of guinea pig. The potassium channel opening effect of fennel may contribute on its relaxant effect on guinea pig tracheal chains [111]. Moreover, anethole bears a striking resemblance to the catecholamines epinephrine, norepinephrine, and dopamine. This structural similarity appears to be responsible for the various sympathomimetic activities of $F$. vulgare such as bronchodilatory effect [71].

4.32. Antioxidant Activities. Naturally occurring antioxidants can be used to protect human beings from oxidative stress damage [112]. Fennel was known as excellent source of natural antioxidants and contributed to the daily antioxidant diet [113]. Wild fennel was found to exhibit a free radical scavenging activity with higher content phenolic and flavonoid than medicinal and edible fennel, and the aerial parts of the Italian fennel populations showed the highest $\mathrm{DPPH}$ scavenging activity [65]. Phenolic compounds of fennel, including caffeoylquinic acid, rosmarinic acid, eriodictyol7-orutinoside, quercetin-3-O-galactoside, and kaempferol-3$O$-glucoside, showed antioxidant activities [67]. The volatile oil showed strong antioxidant activity in comparison with butyrated hydroxyanisole and butylated hydroxytoluene. Ethanol and water extracts of fennel showed less antioxidant activity compared with essential oil [114].

\section{Environmental Application}

Foeniculum vulgare, that is, fennel, not only exhibited pharmacological activities but also revealed a few environmental activities. These activities play a key role in the management of nematode, insect, mosquitoes, and some harmful larvae of malaria producing vector. Thus, the extracts of $F$. vulgare and isolated biologically active compounds have been evaluated for their insecticidal, repellent, acaricidal, larvicidal, and nematicidal activity [115-119]. A brief review on the different type of ecofriendly environmental activities as reported on this plant is summarized below.

5.1. Insecticidal Activities. The fruit derived phytoconstituents of F. vulgare exhibited prominent insecticidal activities against Sitophilus oryzae, Callosobruchus chinensis, and Lasioderma serricorne. This activity was examined using direct contact application and fumigation methods. The biologically active constituents, that is, phenylpropenes (E)anethole and estragole, and the monoterpene $(+)$-fenchone were characterized from Foeniculum fruit. By using a filter 
paper diffusion test, estragole $\left(0.168 \mathrm{mg} \mathrm{cm}^{-2}\right)$ caused $91 \%$ mortality to $S$. oryzae within 1 day after treatment whereas (+)-fenchone and (E)-anethole gave over $90 \%$ mortality at 2 and 4 day after treatment, respectively.

After 2 days of treatment, all test compounds (0.021 $\mathrm{mg} \mathrm{cm}{ }^{-2}$ concentration) revealed potent insecticidal activity against $C$. chinensis. Whereas after 1 day of treatment, (E)anethole $\left(0.105 \mathrm{mg} \mathrm{cm}^{-2}\right)$ gave $100 \%$ mortality of L. serricorne whereas 90 and $60 \%$ mortality at 4 day after treatment was achieved with estragole and (+)-fenchone, respectively. In a fumigation test, the compounds were much more effective against adults of $S$. oryzae, C. chinensis, and L. serricorne in closed cups than in open ones, indicating that the insecticidal activity of test compounds was largely attributable to fumigant action. As naturally occurring insect-control agents, the F. vulgare fruit-derived materials described could be useful for managing field populations of S. oryzae, C. chinensis, and L. serricorne [116].

5.2. Acaricidal Activity. Fennel oil shows significant acaricidal activity against Dermatophagoides farinae and Dermatophagoides pteronyssinus. (+)-fenchone and $\mathrm{p}$-anisaldehyde are major constituents of fruit oil of $F$. vulgare. $P$-anisaldehyde was the most toxic compound against $D$. farinae and is much more effective compared with benzyl benzoate, thymol, and estragol [118].

5.3. Repellent Activity. The methanolic extract of fruits of F. vulgare was spectroscopically characterized for the presence of biologically active constituents called (+)-fenchone and (E)-9-octadecenoic acid. The repellent activity of these constituents was tested against hungry Aedes aegypti females with the help of skin and patch tests and compared with that of the commercial repellent agent called N,N-diethylm-toluamide (DEET) and (Z)-9-octadecenoic acid. In a skin test with female mosquitoes (+)-fenchone and (Z)-9octadecenoic acid $\left(0.4 \mathrm{mg} / \mathrm{cm}^{2}\right)$ exhibited moderate repellent activity at $30 \mathrm{~min}$ after treatment, whereas DEET provided $>1 \mathrm{~h}$ of protection against adult mosquitoes at $\left(0.2 \mathrm{mg} / \mathrm{cm}^{2}\right)$. Thus, (+)-Fenchone and (E)-9-octadecenoic acid are potential mosquito repellent agents or lead compounds [117].

5.4. Larvicidal Activity. Plant extracts and oils may act as alternatives to conventional pesticides for malaria vector control. By considering this aspect, Sedaghat et al. [119] investigated the larvicidal activity of essential oils of three plants of Apiaceae family against malaria vector called Anopheles stephensi. The larvicidal activity was evaluated against laboratory-reared larvae by standard method of WHO. The F. vulgare oil was the most effective against $A$. stephensi with LC(50) and LC(90) values of 20.10 and $44.51 \mathrm{ppm}$, respectively [119]. Additionally, the essential oil extracts from leaves, flowers, and roots of $F$. vulgare exhibit noticeable larvicidal activity against fourth-instar larvae of the mosquito Culex pipiens molestus. Terpineol and 1,8-cineole content of $F$. vulgare are the most effective phytoconstituent against Culex pipiens molestus bites offering complete protection for 1.6 and 2 h, respectively [120]. Recently, Zoubiri et al. [57] reported the larvicidal activity of essential oil of fennel seed against Culex pipiens mosquito. Thus, F. vulgare can serve as a natural larvicidal agent.

5.5. Nematicidal Activity. Oka et al. investigated the in vitro nematicidal activity of essential oils extracted from 27 spices and aromatic plants in pot experiments. Twelve of the twenty-seven essential oils immobilized more than $80 \%$ of juveniles of the root-knot nematode Meloidogyne javanica at a concentration of $1000 \mu \mathrm{L} /$ liter. At this concentration, most of these oils also inhibited nematode hatching. Essential oils of Carum carvi, Foeniculum vulgare, Mentha rotundifolia, and Mentha spicata showed the highest nematicidal activity among the in vitro tested oils. In 3-liter pot experiments, nematicidal activity of the essential oils and their components was confirmed at 200 and $150 \mathrm{mg} / \mathrm{kg}$, respectively. The results suggest that the essential oils and their main components may serve as nematicides [115].

\section{Toxicity}

The long history of ethnomedicinal application, with no reports of any serious side effects, suggests that $F$. vulgare could be considered as safe. In most toxicity experiments carried out on F. vulgare, no sign of toxicity was observed. Shah and his coworker in 1991 investigated the detailed toxicity account of ethanolic extract of fennel fruit in experimental mice with respect to acute and 90 days longer term toxicity [121]. In experimentation, Shah and his coworker observed the general symptoms of toxicity and mortality for only $24 \mathrm{~h}$ in acute toxicity. Whereas, in another part of toxicity they studied the effect of fennel extract on mice with 90 days long term treatment. Acute toxicity of ethanolic extract of $F$. vulgare was assessed in 35 mice by using three concentrations, namely, $0.5,1$, and $3 \mathrm{~g} / \mathrm{kg}$ body weight. In this investigation, $F$. vulgare exhibited no signs of toxicity and no mortality was observed upto the dose level $3 \mathrm{~g} / \mathrm{kg}$ body weight. In case of longer term toxicity, ethanolic extract of $F$. vulgare $(100 \mathrm{mg} / \mathrm{kg}$ body weight/day) was given in drinking water of animals (30 male and 30 female mice). All external morphological, haematological, and spermatogenic changes, in addition to body and vital organ weights, were recorded. The extract caused no significant chronic mortality as compared to controls during this investigation. The treated male mice gained significant weight during chronic treatment while a loss or no significant change in weight was noticed in the female mice treated with the same extract. The extracts did not show spermatotoxic effects. Thus, Shah and his coworker concluded that fennel extract is safe based on both acute and/or long term toxicity studies [121]. Additionally, the plant extract in doses of $0.5,1$, and $3 \mathrm{~g} / \mathrm{kg}$ (orally) did not cause any deaths. These doses do not show any type of toxicity against several parameters tested, namely, locomotor activity, bizarre reactions, sensitivity to sound, social interaction, tail posture, aggressive behaviour, ataxia, paralysis, convulsions, tremors, prostration, exophthalmos, pupil size, defecation, salivation, urination, pattern of respiration, nasal discharge, cyanosis, and piloerection. Exceptionally, only the $3 \mathrm{~g} / \mathrm{kg}$ dose 
showed signs of reduced locomotor activity and piloerection. Otherwise, all other parameters were negative [108]. In another experiment of acute toxicity, different solvent extracts, namely, $n$-hexane, methylene chloride, ethyl acetate, and methanol extracts of F. vulgare upto $5.5 \mathrm{~g} / \mathrm{kg}$ concentration, did not revealed any kind of toxicity in mice, LD50 being: $6.75,11.0,6.92$, and $15 \mathrm{~g} / \mathrm{kg}$ for $n$-hexane, methylene chloride, ethyl acetate, and methanol extracts, respectively [68]. The plant extract of F. vulgare was administered orally at a dose of 100, 200, 400, 600, 800, 1000, and $2000 \mathrm{mg} / \mathrm{kg}$ of body weight of mice. Each group of animals was under visual observation for 10 days for the external behavior of neurological toxicity created by plant extract. Even the mice receiving highest dose of $F$. vulgare extract did not show any mortality or toxicity demonstrating the safety profile of the plant extract [89].

The acute oral 50\% LD50 for anethole in rats was found to be $2090 \mathrm{mg} / \mathrm{kg}$. Repeated doses of one-third the LD50 of anethole $(695 \mathrm{mg} / \mathrm{kg})$ given to rat caused mild liver lesions. It would therefore appear that in normal therapeutic dosages anethole would have minimal hepatotoxicity. When anethole was fed to rats daily for one year as $0.25 \%$ of the diet, no hepatic damage was seen [122]. The acute oral LD50 of essential oil in rats is $1326 \mathrm{mg} / \mathrm{kg}$ [76]. The use of F. vulgare essential oil as a remedy for control of primary dysmenorrhea increases concern about its potential teratogenicity due to its estrogen like activity. Evaluation of teratogenicity of essential oil using limb bud mesenchymal cells showed that the essential oil may have toxic effect on fetal cells, but there was no evidence of teratogenicity upto concentration of $9.3 \mathrm{mg} / \mathrm{mL}$ of culture medium [123]. The overall toxicity studies carried out on $F$. vulgare accounts for its safety at the recommended therapeutic doses.

\section{Conclusions}

The available scientific research on Foeniculum vulgare has shown that it is an important medicinal plant used in a wide range of ethnomedical treatments, especially for abdominal pains, antiemetic, aperitif, arthritis, cancer, colic in children, conjunctivitis, constipation, depurative, diarrhea, dieresis, emmenagogue, fever, flatulence, gastralgia, gastritis, insomnia, irritable colon, kidney ailments, as a laxative, leucorrhoea, liver pain, mouth ulcer, and stomachache. This plant has been in use for a long period of time without any documented serious adverse effects. Studies carried out in the past and present indicate that fennel possesses diverse health benefits and are an important constituent of food. Studies have shown that various extracts of fennel possess a range of pharmacological actions, such as antiaging, antiallergic, anticolitic, antihirsutism, anti-inflammatory, antimicrobial and antiviral, antimutagenic, antinociceptive, antipyretic, antispasmodic, antistress, antithrombotic, anxiolytic, apoptotic, cardiovascular, chemomodulatory action, cytoprotection and antitumor, cytotoxicity, diuretic, estrogenic properties, expectorant, galactogenic, gastrointestinal effect, hepatoprotective, human liver cytochrome P450 3A4 inhibitory, hypoglycemic, hypolipidemic, memory-enhancing property, nootropic, and oculohypotensive activity supporting its traditional use. However, the most prominent and the well studied effects are the antimicrobial and antioxidant effects of essential oil of fennel in different experimental models. The observed health benefits may be credited to the presence of the various phytochemicals like volatile compounds, flavonoids, phenolic compounds, fatty acids, and amino acids.

Fennel also contains mineral and trace elements like aluminum, barium, calcium, cadmium, cobalt, chromium, copper, iron, magnesium, manganese, nickel, lead, strontium, and zinc [124]; fat soluble vitamins such as vitamins A, E, and $\mathrm{K}$; water soluble vitamins like ascorbic acid, thiamine, riboflavin, niacin, and pyridoxine; essential amino acids like leucine, isoleucine, phenylalanine, and tryptophane may contribute to the myriad health beneficial effects at least in part.

Most of the pharmacological studies were conducted using uncharacterized crude extracts of fennel. It is difficult to reproduce the results of these studies and pinpoint the bioactive compounds. Hence, there is a need for chemical standardization and bioactivity-guided identification of bioactive compounds. Among several classes of chemical constituents identified in fennel, volatile components of fennel essential oil and phenolic compounds are assumed to be the main bioactive compounds responsible for the majority of its pharmacological effects. However, the vast traditional use and proven pharmacological activities of fennel indicate that an immense scope still exists for its chemical exploration. Future studies should be focused on validating the mechanism of action responsible for the various beneficial effects and also on understanding which plant based compounds are responsible for the reported effects. The required information when available will enhance our knowledge and appreciation for the use of fennel in our daily diet. Also, the outcome of such chemical studies may further expand its existing therapeutic potential.

Thus, there are many areas of research related to this plant that need to be further explored to fully recognize its beneficial effects for society. Factors such as geographical and seasonal variation play an important role in the authentication of the chemical constituents responsible for the activity which also can be an area of interest. Thus, it is incumbent on researchers to fill the huge gap of insufficient knowledge and create awareness among pharmacologists as well as investigators towards providing better medicinal value derived from this plant. This can be fulfilled only by generating interest among the research community through writing of critical appraisals (paper) and extending the interdisciplinary research area to focused studies on Foeniculum vulgare.

\section{Conflict of Interests}

The authors confirm that this paper's content has no conflict of interests.

\section{Acknowledgments}

The authors acknowledge the Indian Council of Medical Research, New Delhi, for providing Centenary Postdoctoral 
Research Fellowship to Dr. Shamkant B. Badgujar. The authors are grateful to Professor V. S. Kanchi (Librarian, Moolji Jaitha College, Jalgaon, India) for his instructive suggestion in the revised version of the paper. The authors would like to thank all the anonymous reviewers for their constructive comments and thoughtful implications on the paper.

\section{References}

[1] J. Hill, The British Herbal: An History of Plants and Trees, Natives of Britain, Cultivated for Use, or, Raised for Beauty, London, UK, 1756.

[2] B. Muckensturm, D. Foechterlen, J. P. Reduron, P. Danton, and M. Hildenbrand, "Phytochemical and chemotaxonomic studies of Foeniculum vulgare," Biochemical Systematics and Ecology, vol. 25, no. 4, pp. 353-358, 1997.

[3] G. J. Kaur and D. S. Arora, "Antibacterial and phytochemical screening of Anethum graveolens, Foeniculum vulgare and Trachyspermum ammi," BMC Complementary and Alternative Medicine, vol. 9, article 30, 2009.

[4] R. Manonmani and V. M. Abdul Khadir, "Antibacterial screening on Foeniculum vulgare Mill," International Journal of Pharma and Bio Sciences, vol. 2, no. 4, pp. 390-394, 2011.

[5] I. E. Orhan, B. Özçelik, M. Kartal, and Y. Kan, "Antimicrobial and antiviral effects of essential oils from selected Umbelliferae and Labiatae plants and individual essential oil components," Turkish Journal of Biology, vol. 36, no. 3, pp. 239-246, 2012.

[6] P. Morales, A. M. Carvalho, M. C. Sánchez-Mata, M. Cámara, M. Molina, and I. C. F. R. Ferreira, "Tocopherol composition and antioxidant activity of Spanish wild vegetables," Genetic Resources and Crop Evolution, vol. 59, no. 5, pp. 851-863, 2012.

[7] A. Dua, G. Garg, and R. Mahajan, "Polyphenols, flavonoids and antimicrobial properties of methanolic extract of fennel (Foeniculum vulgare Miller)," European Journal of Experimental Biology, vol. 3, no. 4, pp. 203-208, 2013.

[8] T. Malini, G. Vanithakumari, N. Megala, S. Anusya, K. Devi, and V. Elango, "Effect of Foeniculum vulgare. Mill seed extract on the genital organs of male and female rats," Indian Journal of Physiology and Pharmacology, vol. 29, no. 1, pp. 21-26, 1985.

[9] H. Ozbek, S. Uğraş, H. Dülger et al., "Hepatoprotective effect of Foeniculum vulgare essential oil," Fitoterapia, vol. 74, no. 3, pp. 317-319, 2003.

[10] M. Oktay, I. Gülçin, and Ö. I. Küfrevioglu, "Determination of in vitro antioxidant activity of fennel (Foeniculum vulgare) seed extracts," LWT-Food Science and Technology, vol. 36, no. 2, pp. 263-271, 2003.

[11] M. Pradhan, S. Sribhuwaneswari, D. Karthikeyan et al., "In-vitro cytoprotection activity of Foeniculum vulgare and Helicteres isora in cultured human blood lymphocytes and antitumour activity against B16F10 melanoma cell line," Research Journal of Pharmacy and Technology, vol. 1, no. 4, pp. 450-452, 2008.

[12] N. A. El-Soud, N. El-Laithy, G. El-Saeed et al., "Antidiabetic activities of Foeniculum vulgare mill. Essential oil in streptozotocin-induced diabetic rats," Macedonian Journal of Medical Sciences, vol. 4, no. 2, pp. 139-146, 2011.

[13] S. Koppula and H. Kumar, "Foeniculum vulgare Mill (Umbelliferae) attenuates stress and improves memory in wister rats," Tropical Journal of Pharmaceutical Research, vol. 12, no. 4, pp. 553-558, 2013.
[14] L. Barros, A. M. Carvalho, and I. C. F. R. Ferreira, "The nutritional composition of fennel (Foeniculum vulgare): shoots, leaves, stems and inflorescences," LWT: Food Science and Technology, vol. 43, no. 5, pp. 814-818, 2010.

[15] C. Garg, S. A. Khan, S. H. Ansari, A. Suman, and M. Garg, "Chemical composition, therapeutic potential and perspectives of Foeniculum vulgare," Pharmacognosy Reviews, vol. 3, no. 6, pp. 346-352, 2009.

[16] W. He and B. Huang, "A review of chemistry and bioactivities of a medicinal spice: Foeniculum vulgare," Journal of Medicinal Plants Research, vol. 5, no. 16, pp. 3595-3600, 2011.

[17] M. A. Rather, B. A. Dar, S. N. Sofi, B. A. Bhat, and M. A. Qurishi, "Foeniculum vulgare: a comprehensive review of its traditional use, phytochemistry, pharmacology, and safety," Arabian Journal of Chemistry, 2012.

[18] N. S. Jamwal, S. Kumar, and A. C. Rana, "Phytochemical and pharmacological review on Foeniculum Vulgare," vol. 4, pp. 327-341, 2013.

[19] S. Grover, C. P. Malik, A. Hora, and H. B. Kushwaha, "Botany, cultivation, chemical constituents and genetic diversity in fennel (Foeniculum vulgare Mill): a review," International Journal of Life Sciences, vol. 2, no. 2, pp. 128-139.

[20] R. Rahimi and M. R. S. Ardekani, "Medicinal properties of Foeniculum vulgare Mill. in traditional Iranian medicine and modern phytotherapy," Chinese Journal of Integrative Medicine, vol. 19, no. 1, pp. 73-79, 2013.

[21] K. H. Krishnamurthy, "Medicinal plants: Madhurikā, saunf or fennel (Foeniculum vulgare, Gaertn)," Journal of New Approaches to Medicine and Health, vol. 19, no. 1, pp. 1-4, 2011.

[22] C. I. Vardavas, D. Majchrzak, K. H. Wagner, I. Elmadfa, and A. Kafatos, "Lipid concentrations of wild edible greens in Crete," Food Chemistry, vol. 99, no. 4, pp. 822-834, 2006.

[23] M. H. H. Roby, M. A. Sarhan, K. A. Selim, and K. I. Khalel, "Antioxidant and antimicrobial activities of essential oil and extracts of fennel (Foeniculum vulgare L.) and chamomile (Matricaria chamomilla L.)," Industrial Crops and Products, vol. 44, pp. 437-445, 2013.

[24] A. Ghorbani, "Studies on pharmaceutical ethnobotany in the region of Turkmen Sahra, north of Iran (part 1): general results," Journal of Ethnopharmacology, vol. 102, no. 1, pp. 58-68, 2005.

[25] A. Jabbar, M. A. Raza, Z. Iqbal, and M. N. Khan, "An inventory of the ethnobotanicals used as anthelmintics in the Southern Punjab (Pakistan)," Journal of Ethnopharmacology, vol. 108, no. 1, pp. 152-154, 2006.

[26] A. Jain, S. S. Katewa, P. K. Galav, and A. Nag, "Unrecorded ethnomedicinal uses of biodiversity from Tadgarh-Raoli wildlife sanctuary," Acta Botanica Yunnanica, vol. 29, no. 3, pp. 337-344, 2007.

[27] V. B. E. Wyk, "A review of Khoi-San and Cape Dutch medical ethnobotany," Journal of Ethnopharmacology, vol. 119, no. 3, pp. 331-341, 2008.

[28] J. M. Neves, C. Matos, C. Moutinho, G. Queiroz, and L. R. Gomes, "Ethnopharmacological notes about ancient uses of medicinal plants in Trás-os-Montes (northern of Portugal)," Journal of Ethnopharmacology, vol. 124, no. 2, pp. 270-283, 2009.

[29] G. Benítez, M. R. González-Tejero, and J. Molero-Mesa, "Pharmaceutical ethnobotany in the western part of Granada province (southern Spain): ethnopharmacological synthesis," Journal of Ethnopharmacology, vol. 129, no. 1, pp. 87-105, 2010.

[30] V. Savo, C. Giulia, G. P. Maria, and R. David, "Folk phytotherapy of the Amalfi Coast (Campania, Southern Italy)," Journal of Ethnopharmacology, vol. 135, no. 2, pp. 376-392, 2011. 
[31] S. G. D. Oliveira, F. R. R. de Moura, F. F. Demarco, P. D. S. Nascente, F. A. B. D. Pino, and R. G. Lund, "An ethnomedicinal survey on phytotherapy with professionals and patients from Basic Care Units in the Brazilian Unified Health System," Journal of Ethnopharmacology, vol. 140, no. 2, pp. 428-437, 2012.

[32] P. M. Guarrera and V. Savo, "Perceived health properties of wild and cultivated food plants in local and popular traditions of Italy: a review," Journal of Ethnopharmacology, vol. 146, no. 3, pp. 659-680, 2013.

[33] P. M. Guarrera, G. Forti, and S. Marignoli, "Ethnobotanical and ethnomedicinal uses of plants in the district of Acquapendente (Latium, Central Italy)," Journal of Ethnopharmacology, vol. 96, no. 3, pp. 429-444, 2005.

[34] M. J. Macía, E. García, and P. J. Vidaurre, "An ethnobotanical survey of medicinal plants commercialized in the markets of la Paz and El Alto, Bolivia," Journal of Ethnopharmacology, vol. 97, no. 2, pp. 337-350, 2005.

[35] S. Jarić, Z. Popović, M. Macukanović-Jocić et al., "An ethnobotanical study on the usage of wild medicinal herbs from Kopaonik Mountain (Central Serbia)," Journal of Ethnopharmacology, vol. 111, no. 1, pp. 160-175, 2007.

[36] M. Kumar, Y. Paul, and V. K. Anand, "An ethnobotanical study of medicinal plants used by the locals in Kishtwar, Jammu and Kashmir, India," Ethnobotanical Leaflets, vol. 13, no. 10, pp. 12401256, 2009.

[37] F. B. Lewu and A. J. Afolayan, "Ethnomedicine in South Africa: the role of weedy species," African Journal of Biotechnology, vol. 8, no. 6, pp. 929-934, 2009.

[38] S. Mitra and S. K. Mukherjee, "Ethnomedicinal usages of some wild plants of North Bengal plain for gastro-intestinal problems," Indian Journal of Traditional Knowledge, vol. 9, no. 4, pp. 705-712, 2010.

[39] M. Alzweiri, A. A. Sarhan, K. Mansi, M. Hudaib, and T. Aburjai, "Ethnopharmacological survey of medicinal herbs in Jordan, the Northern Badia region," Journal of Ethnopharmacology, vol. 137, no. 1, pp. 27-35, 2011.

[40] M. I. Calvo, S. Akerreta, and R. Y. Cavero, "Pharmaceutical ethnobotany in the Riverside of Navarra (Iberian Peninsula)," Journal of Ethnopharmacology, vol. 135, no. 1, pp. 22-33, 2011.

[41] A. Mesfin, M. Giday, A. Animut, and T. Teklehaymanot, "Ethnobotanical study of antimalarial plants in Shinile District, Somali Region, Ethiopia, and in vivo evaluation of selected ones against Plasmodium berghei," Journal of Ethnopharmacology, vol. 139, no. 1, pp. 221-227, 2012.

[42] R. A. Halberstein, "Botanical medicines for diuresis: crosscultural comparisons," Studies in Natural Products Chemistry, vol. 37, pp. 1-41, 2012.

[43] G. Bulut and E. Tuzlaci, "An ethnobotanical study of medicinal plants in Turgutlu (Manisa-Turkey)," Journal of Ethnopharmacology, vol. 149, no. 3, pp. 633-647, 2013.

[44] M. D. C. Juárez-Vázquez, C. Carranza-Álvarez, A. J. AlonsoCastro et al., "Ethnobotany of medicinal plants used in Xalpatlahuac, Guerrero, México," Journal of Ethnopharmacology, vol. 148 , no. 2, pp. 521-527, 2013.

[45] L. Cornara, A. La Rocca, S. Marsili, and M. G. Mariotti, "Traditional uses of plants in the Eastern Riviera (Liguria, Italy)," Journal of Ethnopharmacology, vol. 125, no. 1, pp. 16-30, 2009.

[46] R. Sharma, R. K. Manhas, and R. Magotra, "Ethnoveterinary remedies of diseases among milk yielding animals in Kathua, Jammu and Kashmir, India," Journal of Ethnopharmacology, vol. 141, no. 1, pp. 265-272, 2012.
[47] E. Carrió and J. Vallès, "Ethnobotany of medicinal plants used in Eastern Mallorca (Balearic Islands, Mediterranean Sea)," Journal of Ethnopharmacology, vol. 141, no. 3, pp. 1021-1040, 2012.

[48] K. R. Kirtikar and B. D. Basu, Indian Medicinal Plants, Vol. IIV, Bishen Singh Mahendra Singh, International Book Distributors, Dehra Dun, India.

[49] I. Grae, Nature's Colors-Dyes from Plants, MacMillan, New York, NY, USA, 1974.

[50] A. Rasul, N. Akhtar, B. A. Khan, T. Mahmood, S. Uz Zaman, and H. M. Shoaib Khan, "Formulation development of a cream containing fennel extract: in vivo evaluation for anti-aging effects," Pharmazie, vol. 67, no. 1, pp. 54-58, 2012.

[51] A. Akgül and A. Bayrak, "Comparative volatile oil composition of various parts from Turkish bitter fennel (Foeniculum vulgare var. vulgare)," Food Chemistry, vol. 30, no. 4, pp. 319-323, 1988.

[52] B. Damjanović, Ž. Lepojević, V. Živković, and A. Tolić, "Extraction of fennel (Foeniculum vulgare Mill.) seeds with supercritical $\mathrm{CO}_{2}$ : comparison with hydrodistillation," Food Chemistry, vol. 92, no. 1, pp. 143-149, 2005.

[53] L. Fang, M. Qi, T. Li, Q. Shao, and R. Fu, "Headspace solvent microextraction-gas chromatography-mass spectrometry for the analysis of volatile compounds from Foeniculum vulgare Mill," Journal of Pharmaceutical and Biomedical Analysis, vol. 41, no. 3, pp. 791-797, 2006.

[54] G. Singh, S. Maurya, M. P. de Lampasona, and C. Catalan, "Chemical constituents, antifungal and antioxidative potential of Foeniculum vulgare volatile oil and its acetone extract," Food Control, vol. 17, no. 9, pp. 745-752, 2006.

[55] M. Tognolini, V. Ballabeni, S. Bertoni, R. Bruni, M. Impicciatore, and E. Barocelli, "Protective effect of Foeniculum vulgare essential oil and anethole in an experimental model of thrombosis," Pharmacological Research, vol. 56, no. 3, pp. 254260, 2007.

[56] I. Telci, I. Demirtas, and A. Sahin, "Variation in plant properties and essential oil composition of sweet fennel (Foeniculum vulgare Mill.) fruits during stages of maturity," Industrial Crops and Products, vol. 30, no. 1, pp. 126-130, 2009.

[57] S. Zoubiri, A. Baaliouamer, N. Seba, and N. Chamouni, "Chemical composition and larvicidal activity of Algerian Foeniculum vulgare seed essential oil," Arabian Journal of Chemistry, 2010.

[58] M. C. Díaz-Maroto, M. S. Pérez-Coello, J. Esteban, and J. Sanz, "Comparison of the volatile composition of wild fennel samples (Foeniculum vulgare Mill.) from Central Spain,” Journal of Agricultural and Food Chemistry, vol. 54, no. 18, pp. 68146818, 2006.

[59] M. Gross, E. Lewinsohn, Y. Tadmor et al., "The inheritance of volatile phenylpropenes in bitter fennel (Foeniculum vulgare Mill. var. vulgare, Apiaceae) chemotypes and their distribution within the plant," Biochemical Systematics and Ecology, vol. 37, no. 4, pp. 308-316, 2009.

[60] M. D. Guillén and M. J. Manzanos, "A study of several parts of the plant Foeniculum vulgare as a source of compounds with industrial interest," Food Research International, vol. 29, no. 1, pp. 85-88, 1996.

[61] N. N. Zhaoa, L. Zhoub, Z. L. Liua, S. S. Duc, and Z. W. Dengd, "Evaluation of the toxicity of the essential oils of some common Chinese spices gainst bostrychophila," Food Control, vol. 26, no. 2, pp. 486-490, 2012.

[62] F. Senatore, F. Oliviero, E. Scandolera et al., "Chemical composition, antimicrobial and antioxidant activities of anethole-rich 
oil from leaves of selected varieties of fennel [Foeniculum vulgare Mill. ssp. vulgare var. azoricum (Mill.) Thell]," Fitoterapia, vol. 90, pp. 214-219, 2013.

[63] P. C. Esquivel-Ferriño, J. M. J. Favela-Hernández, E. GarzaGonzález, N. Waksman, M. Y. Ríos, and M. del Rayo CamachoCorona, "Antimycobacterial activity of constituents from Foeniculum Vulgare Var. Dulce grown in Mexico," Molecules, vol. 17, no. 7, pp. 8471-8482, 2012.

[64] W. Diao, Q. Hu, H. Zhang, and J. Xu, "Chemical composition, antibacterial activity and mechanism of action of essential oil from seeds of fennel (Foeniculum vulgare Mill.)," Food Control, vol. 35, no. 1, pp. 109-116, 2014.

[65] M. Faudale, F. Viladomat, J. Bastida, F. Poli, and C. Codina, "Antioxidant activity and phenolic composition of wild, edible, and medicinal fennel from different Mediterranean countries," Journal of Agricultural and Food Chemistry, vol. 56, no. 6, pp. 1912-1920, 2008.

[66] J. Kunzemann and K. Herrmann, "Isolation and identification of flavon(ol)-O-glycosides in caraway (Carum carvi L.), fennel (Foeniculum vulgare Mill.), anise (Pimpinella anisum L.), and coriander (Coriandrum sativum L.), and of flavon-C-glycosides in anise-I. Phenolics of spices," Zeitschrift für LebensmittelUntersuchung und -Forschung, vol. 164, no. 3, pp. 194-200, 1977.

[67] I. Parejo, O. Jauregui, F. Sánchez-Rabaneda, F. Viladomat, J. Bastida, and C. Codina, "Separation and characterization of phenolic compounds in fennel (Foeniculum vulgare) using liquid chromatography-negative electrospray ionization tandem mass spectrometry," Journal of Agricultural and Food Chemistry, vol. 52, no. 12, pp. 3679-3687, 2004.

[68] M. I. Nassar, E. A. Aboutabl, Y. A. Makled, E. A. ElKhrisy, and A. F. Osman, "Secondary metabolites and pharmacology of Foeniculum vulgare Mill. Subsp. Piperitum," Revista Latinoamericana de Química, vol. 38, no. 2, pp. 103-112, 2010.

[69] J. Cherng, W. Chiang, and L. Chiang, "Immunomodulatory activities of common vegetables and spices of Umbelliferae and its related coumarins and flavonoids," Food Chemistry, vol. 106, no. 3, pp. 944-950, 2008.

[70] M. T. M. Ghanem, H. M. A. Radwan, E. M. Mahdy, Y. M. Elkholy, H. D. Hassanein, and A. A. Shahat, "Phenolic compounds from Foeniculum vulgare (Subsp. Piperitum) (Apiaceae) herb and evaluation of hepatoprotective antioxidant activity," Pharmacognosy Research, vol. 4, no. 2, pp. 104-108, 2012.

[71] M. Albert-Puleo, "Fennel and anise as estrogenic agents," Journal of Ethnopharmacology, vol. 2, no. 4, pp. 337-344, 1980.

[72] H. B. Forster, H. Niklas, and S. Lutz, "Antispasmodic effects of some medicinal plants," Planta Medica, vol. 40, no. 4, pp. 309319, 1980.

[73] I. Chakǔrski, M. Matev, A. Koǐchev, I. Angelova, and G. Stefanov, "Treatment of chronic colitis with an herbal combination of Taraxacum officinale, Hipericum perforatum, Melissa officinaliss, Calendula officinalis and Foeniculum vulgare," Internal Diseases, vol. 20, no. 6, pp. 51-54, 1981.

[74] M. Reiter and W. Brandt, "Relaxant effects on tracheal and ileal smooth muscles of the guinea pig," Arzneimittel-Forschung, vol. 35, no. 1A, pp. 408-414, 1985.

[75] A. S. Abdul-Ghani and R. Amin, "The vascular action of aqueous extracts of Foeniculum vulgare leaves," Journal of Ethnopharmacology, vol. 24, no. 2-3, pp. 213-218, 1988.

[76] S. N. Ostad, M. Soodi, M. Shariffzadeh, N. Khorshidi, and H. Marzban, "The effect of fennel essential oil on uterine contraction as a model for dysmenorrhea, pharmacology and toxicology study," Journal of Ethnopharmacology, vol. 76, no. 3, pp. 299-304, 2001.

[77] S. El Bardai, B. Lyoussi, M. Wibo, and N. Morel, "Pharmacological evidence of hypotensive activity of Marrubium vulgare and Foeniculum vulgare in spontaneously hypertensive rat," Clinical and Experimental Hypertension, vol. 23, no. 4, pp. 329-343, 2001.

[78] K. Javidnia, L. Dastgheib, S. M. Samani, and A. Nasiri, "Antihirsutism activity of Fennel (fruits of Foeniculum vulgare) extract: a double-blind placebo controlled study," Phytomedicine, vol. 10, no. 6-7, pp. 455-458, 2003.

[79] E. Choi and J. Hwang, "Antiinflammatory, analgesic and antioxidant activities of the fruit of Foeniculum vulgare," Fitoterapia, vol. 75, no. 6, pp. 557-565, 2004.

[80] H. Joshi and M. Parle, "Cholinergic basis of memorystrengthening effect of Foeniculum vulgare Linn," Journal of Medicinal Food, vol. 9, no. 3, pp. 413-417, 2006.

[81] F. M. Birdane, M. Cemek, Y. O. Birdane, I. Gülçin, and E. Büyükokuroğlu, "Beneficial effects of vulgare ethanol-induced acute gastric mucosal injury in rat," World Journal of Gastroenterology, vol. 13, no. 4, pp. 607-611, 2007.

[82] M. Kaileh, W. Vanden Berghe, E. Boone, T. Essawi, and G. Haegeman, "Screening of indigenous Palestinian medicinal plants for potential anti-inflammatory and cytotoxic activity," Journal of Ethnopharmacology, vol. 113, no. 3, pp. 510-516, 2007.

[83] Subehan, S. F. Zaidi, S. Kadota, and Y. Tezuka, "Inhibition on human liver cytochrome P450 3A4 by constituents of fennel (Foeniculum vulgare): identification and characterization of a mechanism-based inactivator," Journal of Agricultural and Food Chemistry, vol. 55, no. 25, pp. 10162-10167, 2007.

[84] R. Agarwal, S. K. Gupta, S. S. Agrawal, S. Srivastava, and Saxena., "Oculohypotensive effects of vulgare experimental models of glaucoma," Indian Journal of Physiology and Pharmacology, vol. 52, no. 1, pp. 77-83, 2008.

[85] B. Singh and R. K. Kale, "Chemomodulatory action of Foeniculum vulgare (Fennel) on skin and forestomach papillomagenesis, enzymes associated with xenobiotic metabolism and antioxidant status in murine model system," Food and Chemical Toxicology, vol. 46, no. 12, pp. 3842-3850, 2008.

[86] A. Bogucka-Kocka, H. D. Smolarz, and J. Kocki, "Apoptotic activities of ethanol extracts from some Apiaceae on human leukaemia cell lines," Fitoterapia, vol. 79, no. 7-8, pp. 487-497, 2008.

[87] D. Berrington and N. Lall, "Anticancer activity of certain herbs and spices on the cervical epithelial carcinoma (HeLa) cell line," Evidence-based Complementary and Alternative Medicine, vol. 2012, Article ID 564927, 11 pages, 2012.

[88] F. Oulmouden, R. Saïle, N. El Gnaoui, H. Benomar, and M. Lkhider, "Hypolipidemic and anti-atherogenic effect of aqueous extract of fennel (Foeniculum vulgare) extract in an experimental model of atherosclerosis induced by Triton WR1339," European Journal of Scientific Research, vol. 52, no. 1, pp. 91-99, 2011.

[89] R. Naga Kishore, N. Anjaneyulu, M. Naga Ganesh, and N. Sravya, "Evaluation of anxiolytic activity of ethanolic extract of Foeniculum vulgare in mice model," International Journal of Pharmacy and Pharmaceutical Sciences, vol. 4, no. 3, pp. 584586, 2012.

[90] P. Tripathi, R. Tripathi, R. K. Patel, and S. S. Pancholi, "Investigation of antimutagenic potential of Foeniculum vulgare essential oil on cyclophosphamide induced genotoxicity and oxidative stress in mice," Drug and Chemical Toxicology, vol. 36, no. 1, pp. 35-41, 2013. 
[91] B. L. Duško, L. Čomić, and S. Solujić-Sukdolak, "Antibacterial activity of some plants from family Apiaceae in relation to selected phytopathogenic bacteria," Kragujevac Journal of Science, vol. 28, pp. 65-72, 2006.

[92] M. Gulfraz, S. Mehmood, N. Minhas et al., "Composition and antimicrobial properties of essential oil of Foeniculum vulgare," African Journal of Biotechnology, vol. 7, no. 24, pp. 4364-4368, 2008.

[93] N. Shrivastava and R. Bhargava, "Antibacterial potential of selected medicinal plants against Escherichia coli and Staphylococcus aureus," Acta Biologica Indica, vol. 1, no. 1, pp. 133-135, 2012.

[94] M. R. Martins, M. T. Tinoco, A. S. Almeida, and J. CruzMorais, "Chemical composition, antioxidant and antimicrobial properties of three essential oils from Portuguese flora," Journal of Pharmacognosy, vol. 3, no. 3, pp. 39-44, 2012.

[95] M. M. Özcan, J. Chalchat, D. Arslan, A. Ateş, and A. Ünver, "Comparative essential oil composition and antifungal effect of bitter fennel (Foeniculum vulgare ssp. piperitum) fruit oils obtained during different vegetation," Journal of Medicinal Food, vol. 9, no. 4, pp. 552-561, 2006.

[96] F. Anwar, M. Ali, A. I. Hussain, and M. Shahid, "Antioxidant and antimicrobial activities of essential oil and extracts of fennel (Foeniculum vulgare Mill.) seeds from Pakistan," Flavour and Fragrance Journal, vol. 24, no. 4, pp. 170-176, 2009.

[97] S. H. Park and I. Seong, "Antifungal effects of the extracts and essential oils from Foeniculum vulgare and Illicium verum against Candida albicans," Korean Journal of Medical Mycology, vol. 15, no. 4, pp. 157-164, 2010.

[98] N. Thakur, N. Sareen, B. Shama, and K. Jagota, "Studies on in vitro antifungal activity of Foeniculum vulgare Mill. against spoilage fungi," Global Journal of Bio-Science and BioTechnology, vol. 2, no. 3, pp. 427-430, 2013.

[99] H. A. A. Taie, M. M. I. Helal, W. A. Helmy, and H. Amer, "Chemical composition and biological potentials of aqueous extracts of fennel (Foeniculum vulgare L)," Journal of Applied Sciences Research, vol. 9, no. 3, pp. 1759-1767, 2013.

[100] Y. S. Kwon, W. G. Choi, W. J. Kim et al., "Antimicrobial constituents of Foeniculum vulgare," Archives of Pharmacal Research, vol. 25, no. 2, pp. 154-157, 2002.

[101] A. Zellagui, N. Gherraf, A. Elkhateeb et al., "Chemical constituents from algerian foeniculum vulgare aerial parts and evaluation of antimicrobial activity," Journal of the Chilean Chemical Society, vol. 56, no. 3, pp. 759-763, 2011.

[102] H. Kataoka, S. Horiyama, M. Yamaki et al., "Anti-inflammatory and anti-allergic activities of hydroxylamine and related compounds," Biological \& Pharmaceutical Bulletin, vol. 25, no. 11, pp. 1436-1441, 2002.

[103] P. Padma and R. L. Khosa, "Anti-stress agents from natural origin," Journal of Natural Remedies, vol. 2, no. 1, pp. 21-27, 2002.

[104] K. Devi, G. Vanithakumari, S. Anusya, N. Mekala, T. Malini, and V. Elango, "Effect of Foeniculum vulgare seed extract on mammary glands and oviducts of ovariectomised rats," Ancient Science of Life, vol. 5, no. 2, pp. 129-132, 1985.

[105] T. K. Lim, Edible Medicinal and Non-Medicinal Plants, vol. 5, Springer, New York, NY, USA, 2013.

[106] W. Mueller-Limmroth and H. H. Froehlich, "Effect of various phytotherapeutic expectorants on mucociliary transport," Fortschritte der Medizin, vol. 98, no. 3, pp. 95-101, 1980.

[107] A. Caceres, L. M. Giron, and A. M. Martinez, "Diuretic activity of plants used for the treatment of urinary ailments in
Guatemala," Journal of Ethnopharmacology, vol. 19, no. 3, pp. 233-245, 1987.

[108] M. O. Tanira, A. H. Shah, A. Mohsin, A. M. Ageel, and S. Qureshi, "Pharmacological and toxicological investigations on Foeniculum vulgare dried fruit extract in experimental animals," Phytotherapy Research, vol. 10, no. 1, pp. 33-36, 1996.

[109] M. Yoshioka and T. Tamada, "Aromatic factors of anti-platelet aggregation in fennel oil," Biogenic Amines, vol. 19, no. 2, pp. 8996, 2005.

[110] M. M. Al-Harbi, S. Qureshi, M. Raza, M. M. Ahmed, A. B. Giangreco, and A. H. Shah, "Influence of anethole treatment on the tumour induced by Ehrlich ascites carcinoma cells in paw of Swiss albino mice," European Journal of Cancer Prevention, vol. 4, no. 4, pp. 307-318, 1995.

[111] M. H. Boskabady, A. Khatami, and A. Nazari, "Possible mechanism(s) for relaxant effects of Foeniculum vulgare on guinea pig tracheal chains," Pharmazie, vol. 59, no. 7, pp. 561-564, 2004.

[112] A. Scalbert, C. Manach, C. Morand, and C. Remesy, "Dietary of polyphenols and the prevention of diseases," Reviews in Food Science and Nutrition, vol. 45, no. 4, pp. 287-306, 2005.

[113] A. A. Shahat, A. Y. Ibrahim, S. F. Hendawy et al., "Chemical composition, antimicrobial and antioxidant activities of essential oils from organically cultivated fennel cultivars," Molecules, vol. 16, no. 2, pp. 1366-1377, 2011.

[114] M. C. Díaz-Maroto, I. J. Díaz-Maroto Hidalgo, E. SánchezPalomo, and M. S. Pérez-Coello, "Volatile components and key odorants of fennel (Foeniculum vulgare Mill.) and thyme (Thymus vulgaris L.) oil extracts obtained by simultaneous distillation-extraction and supercritical fluid extraction," Journal of Agricultural and Food Chemistry, vol. 53, no. 13, pp. 53855389, 2005.

[115] Y. Oka, S. Nacar, E. Putievsky, U. Ravid, Z. Yaniv, and Y. Spiegel, "Nematicidal activity of essential oils and their components against the root-knot nematode," Phytopathology, vol. 90, no. 7, pp. 710-715, 2000.

[116] D. H. Kim and Y. J. Ahn, "Contact and fumigant activities of constituents of Foeniculum vulgare fruit against three coleopteran stored-product insects," Pest Management Science, vol. 57, no. 3, pp. 301-306, 2001.

[117] D. Kim, S. Kim, K. Chang, and Y. Ahn, "Repellent activity of constituents identified in Foeniculum vulgare fruit against Aedes aegypti (diptera: Culicidae)," Journal of Agricultural and Food Chemistry, vol. 50, no. 24, pp. 6993-6996, 2002.

[118] H. Lee, "Acaricidal activity of constituents identified in Foeniculum vulgare fruit oil against dermatophagoides spp. (Acari: Pyroglyphidae)," Journal of Agricultural and Food Chemistry, vol. 52, no. 10, pp. 2887-2889, 2004.

[119] M. M. Sedaghat, A. Sanei Dehkordi, M. R. Abai et al., "Larvicidal activity of essential oils of apiaceae plants against malaria vector, Anopheles stephensi," Journal of Arthropod-Borne Diseases, vol. 5, no. 2, pp. 51-59, 2011.

[120] A. F. Traboulsi, S. El-Haj, M. Tueni, K. Taoubi, N. A. Nader, and A. Mrad, "Repellency and toxicity of aromatic plant extracts against the mosquito Culex pipiens molestus (Diptera: Culicidae)," Pest Management Science, vol. 61, no. 6, pp. 597604, 2005.

[121] A. H. Shah, S. Qureshi, and A. M. Ageel, "Toxicity studies in mice of ethanol extracts of Foeniculum vulgare fruit and Ruta chalepensis aerial parts," Journal of Ethnopharmacology, vol. 34, no. 2-3, pp. 167-172, 1991.

[122] J. M. Taylor, P. M. Jenner, and W. I. Jones, "A comparison of the toxicity of some allyl, propenyl, and propyl compounds in the 
rat," Toxicology and Applied Pharmacology, vol. 6, no. 4, pp. 378387, 1964.

[123] S. N. Ostad, B. Khakinegad, and O. Sabzevari, "Evaluation of the teratogenicity of fennel essential oil (FEO) on the rat embryo limb buds culture," Toxicology in Vitro, vol. 18, no. 5, pp. 623627, 2004.

[124] G. Q. Xue, Q. Liu, Y. Q. Han, H. G. Wei, and T. Dong, "Determination of thirteen metal elements in the plant Foeniculum vulgare Mill. by flame atomic absorption spectrophotometry," Guang Pu Xue Yu Guang Pu Fen Xi, vol. 26, no. 10, pp. 19351938, 2006.

[125] R. Di Novella, N. Di Novella, L. De Martino, E. Mancini, and V. De Feo, "Traditional plant use in the National Park of Cilento and Vallo di Diano, Campania, Southern, Italy," Journal of Ethnopharmacology, vol. 145, no. 1, pp. 328-342, 2013.

[126] J. Tardío, M. Pardo-de-Santayana, and R. Morales, "Ethnobotanical review of wild edible plants in Spain," Botanical Journal of the Linnean Society, vol. 152, no. 1, pp. 27-71, 2006.

[127] S. Jarić, M. Mitrović, L. Djurdjević et al., "Phytotherapy in medieval Serbian medicine according to the pharmacological manuscripts of the Chilandar Medical Codex (15-16th centuries)," Journal of Ethnopharmacology, vol. 137, no. 1, pp. 601619, 2011.

[128] V. Tene, O. Malagón, P. V. Finzi, G. Vidari, C. Armijos, and T. Zaragoza, "An ethnobotanical survey of medicinal plants used in Loja and Zamora-Chinchipe, Ecuador," Journal of Ethnopharmacology, vol. 111, no. 1, pp. 63-81, 2007.

[129] P. M. Guarrera, G. Salerno, and G. Caneva, "Folk phytotherapeutical plants from Maratea area (Basilicata, Italy)," Journal of Ethnopharmacology, vol. 99, no. 3, pp. 367-378, 2005.

[130] R. Y. Cavero, S. Akerreta, and M. I. Calvo, "Pharmaceutical ethnobotany in the Middle Navarra (Iberian Peninsula)," Journal of Ethnopharmacology, vol. 137, no. 1, pp. 844-855, 2011.

[131] R. Gupta, M. G. Vairale, R. R. Deshmukh, P. R. Chaudhary, and S. R. Wate, "Ethnomedicinal uses of some plants used by Gond tribe of Bhandara district, Maharashtra," Indian Journal of Traditional Knowledge, vol. 9, no. 4, pp. 713-717, 2010.

[132] A. Mahmood, A. Mahmood, R. N. Malik, and Z. K. Shinwari, "Indigenous knowledge of medicinal plants from Gujranwala district, Pakistan," Journal of Ethnopharmacology, vol. 148, no. 2, pp. 714-723, 2013.

[133] U. P. de Albuquerque, P. M. de Medeiros, A. L. S. de Almeida et al., "Medicinal plants of the caatinga (semi-arid) vegetation of NE Brazil: a quantitative approach," Journal of Ethnopharmacology, vol. 114, no. 3, pp. 325-354, 2007.

[134] R. Polat and F. Satil, "An ethnobotanical survey of medicinal plants in Edremit Gulf (Balikesir, Turkey)," Journal of Ethnopharmacology, vol. 139, no. 2, pp. 626-641, 2012.

[135] L. A. Philander, "An ethnobotany of Western Cape Rasta bush medicine," Journal of Ethnopharmacology, vol. 138, no. 2, pp. 578-594, 2011.

[136] B. Šarić-Kundalić, C. Dobeš, V. Klatte-Asselmeyer, and J. Saukel, "Ethnobotanical study on medicinal use of wild and cultivated plants in middle, south and west Bosnia and Herzegovina," Journal of Ethnopharmacology, vol. 131, no. 1, pp. 33-55, 2010.

[137] D. Kumar, A. Kumar, and O. Prakash, "Potential antifertility agents from plants: a comprehensive review," Journal of Ethnopharmacology, vol. 140, no. 1, pp. 1-32, 2012.

[138] P. Bhat, G. Hegde, and G. R. Hegde, "Ethnomedicinal practices in different communities of Uttara Kannada district of Karnataka for treatment of wounds," Journal of Ethnopharmacology, vol. 143, no. 2, pp. 501-514, 2012.
[139] S. de Marino, F. Gala, N. Borbone et al., "Phenolic glycosides from Foeniculum vulgare fruit and evaluation of antioxidative activity," Phytochemistry, vol. 68, no. 13, pp. 1805-1812, 2007.

[140] R. H. Mohamad, A. M. El-Bastawesy, M. G. Abdel-Monem et al., "Antioxidant and anticarcinogenic effects of methanolic extract and volatile oil of fennel seeds (Foeniculum vulgare)," Journal of Medicinal Food, vol. 14, no. 9, pp. 986-1001, 2011.

[141] M. D. R. Camacho-Corona, M. A. Ramírez-Cabrera, O. González-Santiago, E. Garza-González, I. D. P. Palacios, and J. Luna-Herrera, "Activity against drug resistant-tuberculosis strains of plants used in Mexican traditional medicine to treat tuberculosis and other respiratory diseases," Phytotherapy Research, vol. 22, no. 1, pp. 82-85, 2008.

[142] M. B. Stefanini, R. O. Figueiredo, L. C. Ming, and A. F. Júnior, "Antimicrobial activity of the essential oils of some spice herbs," in Proceedings of the 2nd National CE Research Conference, Horticultural Report No. 28, vol. 597, pp. 215-216, 2003, http://www.actahort.org/books/597/597_30.htm.

[143] P. Lo Cantore, N. S. Iacobellis, A. De Marco, F. Capasso, and F. Senatore, "Antibacterial activity of Coriandrum sativum L. and Foeniculum vulgare Miller var. vulgare (miller) essential oils," Journal of Agricultural and Food Chemistry, vol. 52, no. 26, pp. 7862-7866, 2004.

[144] O. Ertürk, T. B. Özbucak, and A. Bayrak, "Antimicrobial activities of some medicinal essential oils," Herba Polonica, vol. 52, no. 1-2, pp. 58-66, 2006.

[145] M. Araque, L. B. Rojas, and A. Usubillaga, "Antimicrobial activities of essential oil of Foeniculum vulgare miller against multiresistant gram negative Bacillus from nosocomial infections," Ciencia, vol. 15, no. 3, pp. 366-370, 2007.

[146] K. F. Abed, "Antimicrobial activity of essential oils of some medicinal plants from Saudi Arabia," Saudi Journal of Biological Sciences, vol. 14, no. 1, pp. 53-60, 2007.

[147] A. Grigore, S. Colceru-Mihul, I. Paraschiv et al., "Chemical analysis and antimicrobial activity of indigenous medicinal species volatile oils," Romanian Biotechnological Letters, vol. 17, no. 5, pp. 7620-7627, 2012.

[148] M. Sajedi, S. Mirzaei, and A. Yazdinezhad, "Evaluation of the chemical composition and antimicrobial activity of different fennel (Foeniculum vulgare Mill.) ecotypes essential oils from Iran," Research in Pharmaceutical Science, vol. 7, no. 5, p. S719, 2012.

[149] S. Purkayastha, R. Narain, and P. Dahiya, "Evaluation of antimicrobial and phytochemical screening of Fennel, Juniper and Kalonji essential oils against multi drug resistant clinical isolates," Asian Pacific Journal of Tropical Biomedicine, vol. 2, no. 3, pp. S1625-S1629, 2012.

[150] D. R. Saumendu, T. Apu, S. Dhrubajyoti, K. Arunav, B. Anupam, and D. Bidyut, "Antimicrobial potential of volatile oil isolated from some traditional Indian spices," International Research Journal of Pharmacy, vol. 3, no. 4, pp. 162-163, 2012.

[151] M. Kazem, E. Mousavi, and H. Kharestani, "Chemical composition and antimicrobial activities of essential oils of Varthemia persica, Foeniculum vulgare and Ferula lycia," Current Research in Bacteriology, vol. 5, no. 2, pp. 42-52, 2012.

[152] J. R. Skrobonja, D. N. Delić, M. A. Karaman, M. N. Matavulj, and M. A. Bogavac, "Antifungal properties of Foeniculum vulgare, Carum carvi and Eucalyptus sp. ssential oils against Candida albicans strains," Journal of Natural Science, vol. 124, pp. 195202, 2013. 

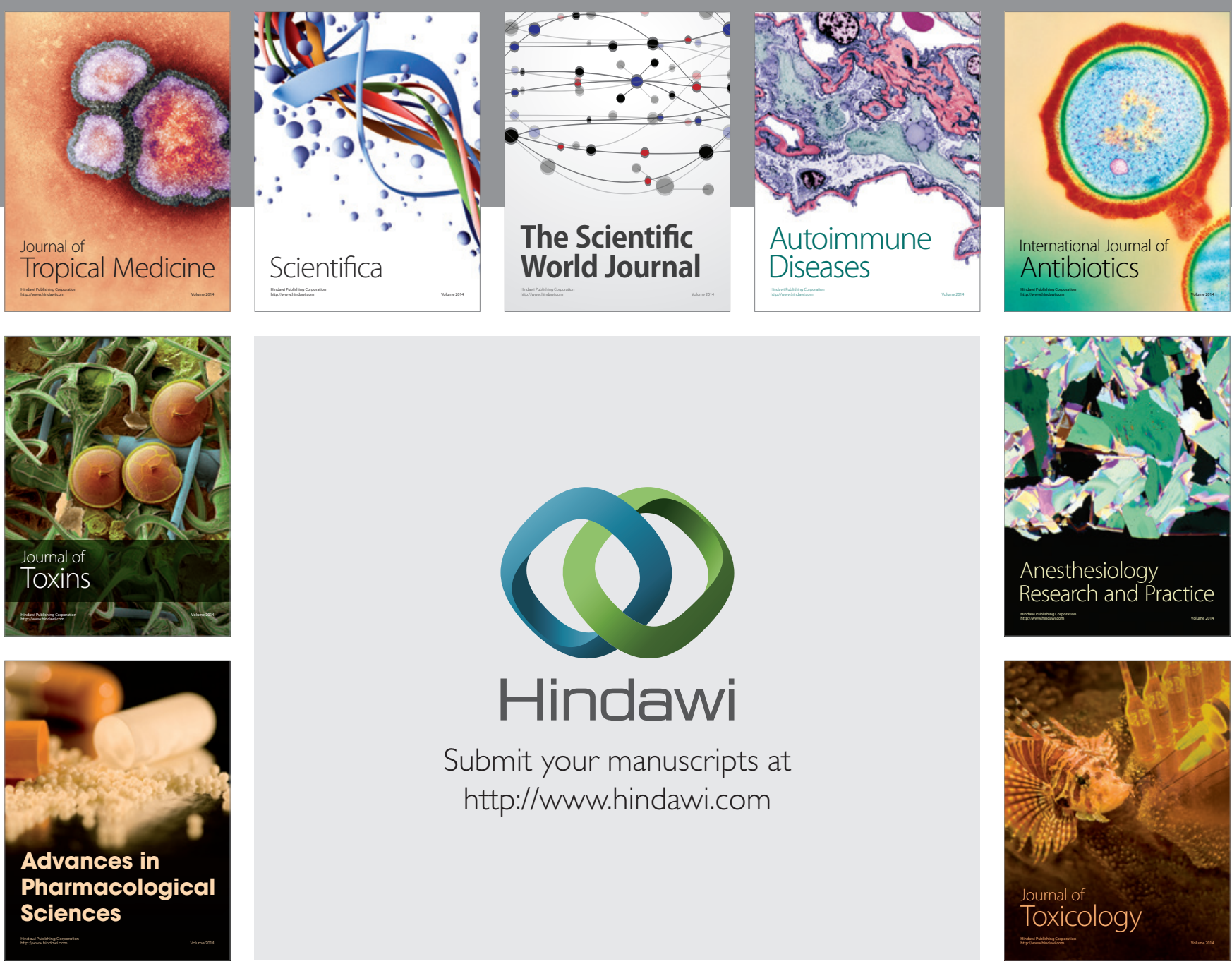

\section{Hindawi}

Submit your manuscripts at

http://www.hindawi.com
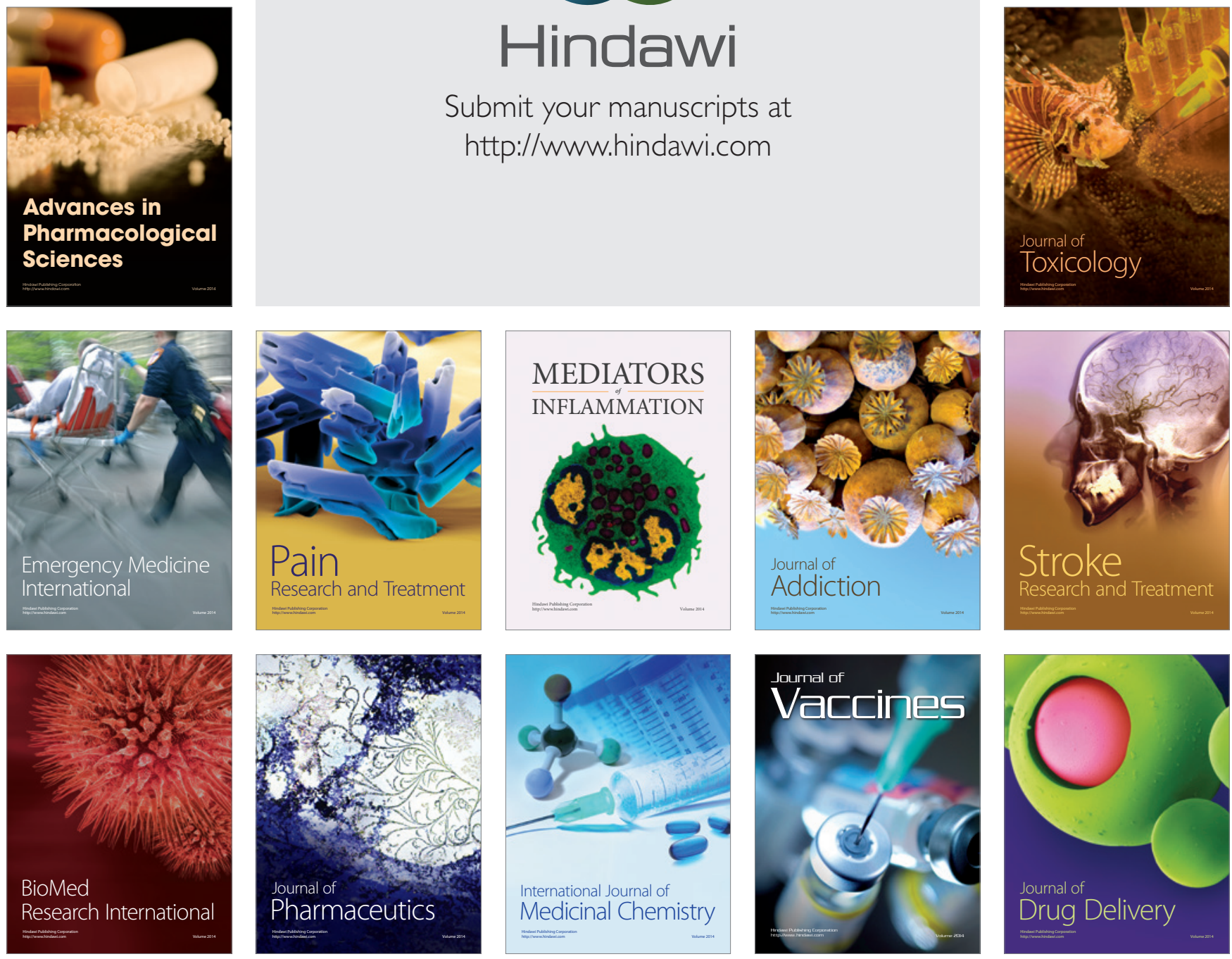